\title{
Warm inflation within a supersymmetric distributed mass model
}

\author{
Mar Bastero-Gil, ${ }^{1, *}$ Arjun Berera, ${ }^{2, \dagger}$ Rafael Hernández-Jiménez, ${ }^{2, \$}$ and João G. Rosa ${ }^{3, \S}$ \\ ${ }^{1}$ Departamento de Física Téorica y del Cosmos, Universidad de Granada, Granada-18071, Spain \\ ${ }^{2}$ School of Physics and Astronomy, University of Edinburgh, Edinburgh, EH9 3FD, United Kingdom \\ ${ }^{3}$ Departamento de Física da Universidade de Aveiro and CIDMA, \\ Campus de Santiago, 3810-183 Aveiro, Portugal
}

(Received 5 February 2019; published 16 May 2019)

\begin{abstract}
We study the dynamics and observational predictions of warm inflation within a supersymmetric distributed mass model. This dissipative mechanism is well described by the interactions between the inflaton and a tower of chiral multiplets with a mass gap, such that different bosonic and fermionic fields become light as the inflaton scans the tower during inflation. We examine inflation for various mass distributions, analyzing in detail the dynamics and observational predictions. We show, in particular, that warm inflation can be consistently realized in this scenario for a broad parametric range and in excellent agreement with the Planck legacy data. Distributed mass models can be viewed as realizations of the landscape property of string theory, with the mass distributions coming from the underlying spectra of the theory, which themselves would be affected by the vacuum of the theory. We discuss the recently proposed swampland criteria for inflation models on the landscape and analyze the conditions under which they can be met within the distributed mass warm inflation scenario. We demonstrate mass distribution models with a range of consistency with the swampland criteria including cases in excellent consistency.
\end{abstract}

DOI: 10.1103/PhysRevD.99.103520

\section{INTRODUCTION}

The most recent cosmological observations once again confirm an expanding universe that is spatially flat, homogeneous, and isotropic on large scales, and where the large scale structure originated from primordial fluctuations with a nearly scale-invariant, adiabatic, and Gaussian spectrum [1]. Inflation [2] remains the dominant paradigm that can consistently explain the observational data. In the standard inflation picture, cold inflation (CI), depicted by a homogeneous scalar "inflaton" field, the short period of quasi-de Sitter accelerated expansion phase quickly dilutes away all traces of any preinflationary matter or radiation density, so that the state of the universe is the vacuum state. However, this generates a supercooled universe and leaves indeterminate a reasonable description of the transition from inflation to the "hot big bang" scenario, required by big bang nucleosynthesis, and the physics of recombination leading to the cosmic microwave background $(\mathrm{CMB})$ that we

\footnotetext{
*mbg@ugr.es

†ab@p.ed.ac.uk

s1367850@sms.ed.ac.uk

joao.rosa@ua.pt
}

Published by the American Physical Society under the terms of the Creative Commons Attribution 4.0 International license. Further distribution of this work must maintain attribution to the author(s) and the published article's title, journal citation, and DOI. Funded by SCOAP. observe today. This necessarily requires the conversion of inflaton energy density into ordinary matter and radiation and thus to its interactions with other fields.

In the conventional inflation picture the inflaton decay can only play a significant role at the end of the slow-roll regime, since particle production is not pictured to occur within the inflationary expansion phase. This leads to cold inflation ending through the standard "(p)reheating" paradigm [3]. The reasoning behind this phase lies in the fact that the perturbative decay width of a particle is generically smaller than its mass, which in turn lies below the Hubble expansion rate for a slowly rolling scalar field. Consequently such interplay between the inflaton and other constituents may perform a negligible role during the slow-roll phase of inflationary models. Nonetheless, it is relevant to note that the perturbative decay width only describes the decay of a field close to the minimum of its potential [4], which is evidently not the case during slow-roll dynamics, and that finite temperature effects can further significantly enhance the rate at which the inflaton dissipates its energy into other degrees of freedom (d.o.f.). Thereby the inflaton field could be coupled to other components and might dissipate its vacuum energy and warm up the universe. This alternative scenario is known as the warm inflation (WI) paradigm $[5,6]$, where dissipative effects and associated particle production can, in fact, sustain a thermal bath concurrently with the accelerated expansion of the universe during inflation. 
One of the earliest warm inflation models [7] suggested the idea that parameters in an inflation model could be randomly distributed. The distributed-mass model (DM model) [8-11] was subsequently proposed and built on this idea in the context of string theory. It observed [9] that models from string theory have states at many energy levels and the distribution of these levels is ultimately dictated by the string vacuum. In particular, depending on the details of the compactification, the state of Kaluza-Klein modes, and patterns of symmetry breaking, it will induce splittings of string energy levels. Thus, based on the specific properties of a given string realization, the string states will be distributed differently. The idea of such distribution of states can find a more dynamical motivation in the context of the landscape picture, whereby different ground states of string theory will in turn imply changes to the spectrum of string states. In this respect, the DM model was one of the first low-energy realizations following the landscape idea well before the idea was formally stated.

The string landscape idea [12,13] claims an unimaginable huge number of possible vacua reaching by some estimates to order $10^{500}$. To have any hope of dealing with such a large number of possibilities, one would need to work from the direction of both string theory and phenomenology to identify viable vacua. The string landscape emerges from the complex structure of string theory. One property of this complex structure is the huge number of string states, going into the hundreds of thousands. Inflation models motivated by the landscape are generally built with only a small number of fields and generally do not attempt to utilize the vast spectra of string states. DM models are one of the few that attempt to utilize as part of the dynamical solution this inherent feature that there are many string states available. In the landscape way of thinking therefore, DM models, although phenomenologically motivated, presumably are studying theories emerging from a vast range of vacua that otherwise are missed in inflation models with just a small number of fields. As such, in constructing DM models it is natural to look for distributions that are of observational interest. In this paper we will first look at the generic DM distribution, where the string states in the range relevant to inflation are equally spaced apart, which was the original example studied in the early work [8-11]. This case has many relevant features but we find that it is not consistent with present observational constraints. We will then examine other types of DM distribution that have appealing consequences in comparison with observation.

Inflation is assumed to be described by low-energy effective field theory (EFT), although in many models inflation can happen when the inflaton field is superPlanckian, particularly by considering monomial chaotic potentials in cold inflation. Furthermore, an EFT can be ultraviolet (UV) complete if it can successfully be incorporated in a quantum theory of gravity, such as string theory.
This paradigm provides a vast landscape of consistent embeddings of the EFT of gravity into a quantum theory, but this does not imply that any EFT coupled to gravity is consequently included in the landscapes. Those EFTs that are, in fact, inconsistent with a quantum theory of gravity lie in the surrounding swamplands [14,15]. Hence, a benchmark is needed to ensure a de Sitter vacuum EFT can live in the desired string landscapes. Recently two swampland criteria relevant for inflationary theories have been proposed [16,17], $|\Delta \phi| / M_{P}<\Delta$ and $M_{P}\left|V_{\phi}\right| / V>c$, provided that $V>0$, where $\{\Delta, c\} \sim \mathcal{O}(1)$. Nonetheless, these criteria have been noted to pose inherent threats to the basic mechanism of slow roll in cold inflation [17]. However, as part of the subsequent analysis we evaluate the aforementioned criteria in the warm inflation scenario. We show models of warm inflation that can be very consistent with the swampland criteria. Inherently the dissipative feature in warm inflation makes it amenable for consistency with swampland criteria, as already noted in the literature [18-22].

In this paper we will examine in detail DM models and explore their consistency with observational data and theoretical viability. This work is organized as follows. In Sec. II we introduce all WI dynamics and the primordial perturbation spectrum. In Sec. III we present in detail the supersymmetric distributed mass model, which is described by the interactions between the inflaton field and other light constituents: fermion $\left(\left\{\psi_{i}, \psi_{\sigma}\right\}\right)$ and scalar $\left(\left\{\chi_{i}, \sigma\right\}\right)$ fields. In Sec. IV we calculate all relevant parameters of the dissipation dynamics for the bosonic and fermionic sectors: dissipative coefficients and their corresponding thermal averages of the decay width. In Sec. V we develop a form of the mass distribution function. In Sec. VI we analyze in detail various dissipative coefficients for several inflation driven monomial potentials; we apply standard slow-roll methods and identify observationally consistent regions in parameter space. Here we also test the recently proposed swampland criteria. The main conclusions of this work are summarized in Sec. VII. Three Appendixes are also included, where we provide more detailed discussions of some of the results used in our computations.

\section{WARM INFLATION DYNAMICS AND PRIMORDIAL PERTURBATION SPECTRUM}

Nonequilibrium effects in the dynamics of a scalar field are generically produced due to interactions with an ambient thermal bath. The leading nonequilibrium effect, for a field evolving slowly compared to the characteristic timescale of the thermal bath, is a dissipative friction term $\Upsilon \dot{\phi}$ in its equation of motion [23], where $\Upsilon=\Upsilon(\phi, T)$ can be computed from first principles given the form of the interactions between the scalar field and the thermalized d.o.f. For a homogeneous field, this implies the continuity equation $\dot{\rho}_{\phi}+3 H\left(\rho_{\phi}+p_{\phi}\right)=-\Upsilon \dot{\phi}^{2}$, such that overall 
energy-momentum conservation implies the existence of an identical term with the opposite sign in the continuity equation for the thermal fluid. This explicitly proves that dissipative effects in the inflaton's equation of motion lead to particle production in the thermal bath, so it prevents the exponential dilution of the latter in a quasi-de Sitter background. Therefore the temperature does not drop abruptly, and the universe is able to smoothly cross to the radiation epoch. Taking into account dissipative effects, the evolution equation for the background inflaton field is given by

$$
\ddot{\phi}+(3 H+\Upsilon) \dot{\phi}+V_{\mathrm{eff}, \phi}(\phi, T)=0,
$$

where $V_{\text {eff, } \phi}=d V_{\text {eff }} / d \phi=V(\phi)_{, \phi}+V_{T, \phi}$ includes the effective thermal potential $V_{\text {eff }}=V(\phi)+V_{T}$, where $V(\phi)$ is the zero temperature potential, and $V_{T}$ is the finite temperature effective potential, including radiative corrections to the potential due to any light component (fermion or scalar fields); $\Upsilon$ is the dissipative coefficient and $H$ is the Hubble parameter, given by the Friedmann equation for a flat Friedmann-Roberston-Walker universe:

$$
H^{2}=\frac{\rho_{T}}{3 M_{P}^{2}},
$$

where $\rho_{T}=\dot{\phi}^{2} / 2+V_{\text {eff }}+T s_{R}$ is the total energy density of the system, $s_{R}$ is the entropy density, and $M_{P}$ is the reduced Planck mass. One parameter that quantifies dissipation during WI is defined as the dissipative ratio $Q=\Upsilon /(3 H)$. Depending on the ratio $Q$ we can have different regimes: when $Q<1$, this is called weak dissipative warm inflation; and when $Q \gtrsim 1$, we are in strong dissipative warm inflation. Furthermore, the WI paradigm assumes the presence of a thermal bath, at temperature $T$; hence the evolution of such a dissipative mechanism can be obtained from the evolution equation for the entropy density, given by

$$
T\left(\dot{s}_{R}+3 H s_{R}\right)=\Upsilon \dot{\phi}^{2},
$$

which in the slow-roll regime reduces to $3 H T s_{R} \simeq \Upsilon \dot{\phi}^{2}$. Without including the $T$-dependent corrections in the inflaton potential, we would have the standard relation $T s_{R}=4 \rho_{R} / 3=4 C_{R} T^{4} / 3$, where $C_{R}=\pi^{2} g_{\mathrm{eff}}(T) / 30$, $g_{\text {eff }}(T)$ is the effective $T$-dependent number of relativistic d.o.f., with $\rho_{R}$ denoting the standard energy density of radiation. All relativistic light fields contribute to the effective d.o.f., having

$$
g_{\mathrm{eff}}(T)=\frac{90}{4 \pi^{2}} \frac{s_{R}}{T^{3}} .
$$

Since the radiative corrections modify the potential and its derivatives, they alter the standard slow-roll parameters, being from $\epsilon_{\phi}=M_{P}^{2}\left(V_{, \phi} / V\right)^{2} / 2$ and $\eta_{\phi}=M_{P}^{2} V_{, \phi \phi} / V$ to

$$
\epsilon_{\mathrm{eff}}=\frac{M_{p}^{2}}{2}\left(\frac{V_{\mathrm{eff}, \phi}}{V_{\mathrm{eff}}}\right)^{2}, \quad \eta_{\mathrm{eff}}=M_{p}^{2} \frac{V_{\mathrm{eff}, \phi \phi}}{V_{\mathrm{eff}}}
$$

where $V_{\text {eff, } \phi \phi}=V(\phi)_{, \phi \phi}+V_{T, \phi \phi}$. Recall that inflation happens when $\dot{\phi}^{2} / 2 \ll V_{\text {eff }}$ and so does $\left(T s_{R} Q^{-1} / 2\right) \ll V_{\text {eff }}$, but even if it is small compared to the inflaton effective potential, it can be larger than the expansion rate with $\left(T s_{R}\right)^{1 / 4} \gtrsim H$; by assuming thermalization, this translates roughly into $T \gtrsim H$, so one can consistently obtain a warm inflationary universe with a slow-roll evolution, as long as the radiative corrections are restrained such they do not spoil inflation. Furthermore, one can show that the radiation energy density portrayed by an entropic description can never exceed the inflationary potential in a slow-roll regime, guaranteeing a period of accelerated expansion,

$$
\frac{T s_{R}}{V_{\mathrm{eff}}} \simeq \frac{2}{3} \frac{\epsilon_{\mathrm{eff}}}{1+Q} \frac{Q}{1+Q},
$$

such that consistency of the slow-roll evolution requires $\epsilon_{\text {eff }}<1+Q$. This in turn also implies that, at the end of the slow-roll regime, when $\epsilon_{\text {eff }} \sim 1+Q$, one may attain $T s_{R} \sim$ $V_{\text {eff }}$ if a strong dissipative regime $Q \gtrsim 1$ can be achieved. In such cases radiation will smoothly become the dominant component at the end of inflation, providing the necessary "graceful exit" into the hot big bang cosmic evolution [24]. Although there may be additional particle production at the end of inflation, no reheating is actually necessary in WI when strong dissipation is reached; otherwise, such a mechanism is needed. In addition to the smooth exit from inflation, WI exhibits several attractive features that have been explored in recent years. For instance, the dissipative friction damps the inflaton's evolution, making slow roll easier or, equivalently, alleviating the conditions on the flatness of the inflaton potential, expressed now by the slow-roll conditions $\epsilon_{\text {eff }},\left|\eta_{\text {eff }}\right| \ll 1+Q$. This may potentially provide a solution to the so-called "eta problem" typically found in string/supergravity inflationary models where generically $\eta_{\phi} \sim \mathcal{O}(1)$ [10,25]. (For other recent reviews of warm inflation, please see $[26,27]$.)

Small fluctuations of the inflaton about its homogenous component provide the initial seeds of density perturbation. These density perturbations produced during inflation evolve into the classical inhomogeneities observed in the CMB. For WI scenarios the fluctuations of the inflaton are thermally induced. As such, these initial seeds of density perturbations are already classical upon definition. The general expression for the amplitude of the primordial spectrum is given by $[4,28-31]$

$\Delta_{\mathcal{R}}^{2}=\left(\frac{H_{*}}{\dot{\phi}_{*}}\right)^{2}\left(\frac{H_{*}}{2 \pi}\right)^{2}\left(1+2 n_{*}+\frac{2 \sqrt{3} \pi Q_{*}}{\sqrt{3+4 \pi Q_{*}}} \frac{T}{H}\right) G\left(Q_{*}\right)$, 
where all quantities are evaluated when the relevant CMB modes become superhorizon 50-60 e-folds before inflation ends. In the expression above, $n_{*}$ denotes the inflaton phase space distribution at the horizon crossing. By the strength of the interactions between the inflaton field and other particles in the thermal bath (including, e.g., scattering processes), this might interpolate between the BunchDavies vacuum, $n_{*}=0$, and the Bose-Einstein distribution at the ambient temperature $T, n_{*} \simeq\left(e^{H_{*} / T_{*}}-1\right)^{-1}$. We will focus on the latter limiting case in this paper, which we denote as "thermal" inflaton fluctuations. Also the function $G\left(Q_{*}\right)$ accounts for the growth of inflaton fluctuations due to the coupling to radiation fluctuations through the temperature dependence of the dissipation coefficient and must be determined numerically. In addition, this function also exhibits a mild dependence on the form of the scalar potential. In general, with thermalized inflation fluctuations $1+2 n_{*}=\operatorname{coth}\left(H_{*} /\left(2 T_{*}\right)\right)$, we have

$$
\begin{aligned}
\Delta_{\mathcal{R}}^{2} \simeq & \left(\frac{3 H_{*}^{3}\left(1+Q_{*}\right)}{2 \pi V_{\mathrm{eff}, \phi *}}\right)^{2} \\
& \times\left(\frac{2 \sqrt{3} \pi Q_{*}}{\sqrt{3+4 \pi Q_{*}}} \frac{T_{*}}{H_{*}}+\operatorname{coth}\left(\frac{H_{*}}{2 T_{*}}\right)\right) G\left(Q_{*}\right) ;
\end{aligned}
$$

then from the amplitude of the curvature power spectrum, we may determine the scalar spectral index $n_{s}-1 \simeq d \ln \Delta_{\mathcal{R}}^{2} / d N_{e}$. Since, for $T \ll M_{P}$, gravitational waves are not significantly affected by thermal effects, the primordial tensor spectrum is given by the standard inflationary form $\Delta_{t}^{2}=2 H_{*}^{2} /\left(\pi^{2} M_{P}^{2}\right)$. The tensor-to-scalar ratio $r=\Delta_{t}^{2} / \Delta_{\mathcal{R}}^{2}$ is nevertheless affected, and, in fact, is typically reduced, by the modifications to the scalar curvature perturbations introduced due to dissipation, which are basically a function of $T_{*} / H_{*}$ and $Q_{*}$. We illustrate this fact by using the slow-roll dynamics, where the ratio $r$ can be written as

$$
\begin{aligned}
r & \simeq \frac{16 \epsilon_{\mathrm{eff}}}{\left(1+Q_{*}\right)^{2} F\left(T_{*} / H_{*}, Q_{*}\right)}, \\
F\left(T_{*} / H_{*}, Q_{*}\right) & =\left(\frac{2 \sqrt{3} \pi Q_{*}}{\sqrt{3+4 \pi Q_{*}}} \frac{T_{*}}{H_{*}}+\operatorname{coth}\left(\frac{H_{*}}{2 T_{*}}\right)\right) G\left(Q_{*}\right),
\end{aligned}
$$

which is suppressed with respect to the CI prediction by a factor $\left(1+Q_{*}\right)^{2} K\left(T_{*} / H_{*}, Q_{*}\right)>1$. Indeed, in [25] it was shown explicitly, even before the BICEP and Planck results, that the presence of radiation and dissipation suppresses the tensor-to-scalar ratio. Authors in [25] computed the tensorto-scalar ratio of the monomial $\phi^{2}$ and $\phi^{4}$ models, and it was one of the few analyses at the time that predicted for these inflation driven potentials a low tensor-to-scalar ratio, which now we see is consistent with data. Subsequent work further developed the analysis [32-36], showing that the tensor-to-scalar ratio may attain values even below $10^{-3}$ for a $\phi^{4}$ potential, and thus potentially distinguishable from, e.g., scenarios with a nonminimal coupling to gravity such as Higgs inflation [37] or the Starobinsky model [2].

\section{SUPERSYMMETRIC DISTRIBUTED MASS MODEL}

Let us consider the general form of an effective $\mathrm{N}=1$ global supersymmetry (SUSY) theory version of the distributed mass model with chiral superfields $\Phi, X_{i}$, and $Y_{i}$, described by the superpotential [25,38-40]

$$
W=\sum_{i}\left[\frac{g}{2}\left(\Phi-M_{i}\right) X_{i}^{2}+\frac{h}{2} X_{i} Y_{i}^{2}\right],
$$

where $g$ and $h$ are coupling constants and the sum is taken over an arbitrary distribution of supermultiplets $X_{i}$ and $Y_{i}$. The chiral superfields $\Phi, X_{i}$, and $Y_{i}$ have (scalar, fermion) components $\left(\phi, \psi_{\phi}\right),\left(\chi_{i}, \psi_{\chi_{i}}\right)$, and $\left(\sigma_{i}, \psi_{\sigma_{i}}\right)$, respectively. Note that these are complex scalars and Weyl fermions, each with 2 d.o.f. We may use the Majorana representation for the spinors, i.e., use a four-component Majorana spinor built from the same Weyl fermion. In this case, note that a Majorana fermion is its own antiparticle. We have considered different $Y_{i}$ fields coupled to each $X_{i}$ field in the tower to avoid mass mixing at the level of the thermal masses. The scalar interaction terms in the theory are obtained from the superpotential using [38]

$$
-\mathcal{L}_{S}=\left|\partial_{\Phi} W\right|^{2}+\sum_{i}\left|\partial_{X_{i}} W\right|^{2}+\sum_{i}\left|\partial_{Y_{i}} W\right|^{2} .
$$

On the other hand, the fermion Lagrangian can be computed from the general formula [38]

$-\mathcal{L}_{F}=\frac{1}{2} \sum_{n, m} \frac{\partial^{2} W}{\partial \xi_{n} \partial \xi_{m}} \bar{\psi}_{n} P_{L} \psi_{m}+\frac{1}{2} \sum_{n, m} \frac{\partial^{2} W^{\dagger}}{\partial \xi_{n}^{\dagger} \partial \xi_{m}^{\dagger}} \bar{\psi}_{n} P_{R} \psi_{m}$,

where $\xi_{n}$ is a superfield: $\Phi, X_{i}, Y_{i}$, and $P_{L}=1-P_{R}=$ $\left(1+\gamma_{5}\right) / 2$ are the chiral projection operators acting on Majorana four-spinors. Note that $\partial W / \partial X_{i} \partial Y_{i}=$ $\partial W / \partial Y_{i} \partial X_{i}$ and similarly $\partial W^{\dagger} / \partial X_{i}^{\dagger} \partial Y_{i}^{\dagger}=\partial W^{\dagger} / \partial Y_{i}^{\dagger} \partial X_{i}^{\dagger}$. Then we select only the boson field components of the chiral superfields $\Phi, X_{i}$, and $Y_{i}$, which are $\phi, \chi_{i}$, and $\sigma_{i}$, respectively. Nonetheless, see that the inflaton field $\phi$ can be decomposed into its real and imaginary parts via $^{1}$ $\phi=\left(\phi_{R}+i \phi_{I}\right) / \sqrt{2}$. Hence, this prescription introduces another decay channel; for instance, the modulus square becomes $\left|\phi-M_{i}\right|^{2}=\left(\phi_{R} / \sqrt{2}-M_{i}\right)^{2}+\phi_{I}^{2} / 2$. However, $\phi_{R} / \sqrt{2}$ is the nonzero vacuum expectation value of $\phi$, which will be the only term that will contribute to dissipation in the scalar field's effective equation of motion,

\footnotetext{
${ }^{1}$ Similarly we define the complex scalars in terms of the real and imaginary parts for $\chi_{i}$ and $\sigma_{i}$.
} 
so that the imaginary part of the inflaton, $\phi_{I}$, is not relevant for our subsequent analysis. Moreover, in order to be coherent with further calculations, $\phi$ is going to be considered only as the classical expectation value, without the label $R$ and the factor $1 / \sqrt{2}$. Therefore, the relevant Lagrangians that may contribute to dissipation in the scalar field's effective equation of motion are

$$
\begin{aligned}
-\mathcal{L}_{S}= & g^{2} \sum_{i}\left(\phi-M_{i}\right)^{2}\left|\chi_{i}\right|^{2}+\frac{g h}{2} \sum_{i}\left(\phi-M_{i}\right)\left[\chi_{i}\left(\sigma_{i}^{\dagger}\right)^{2}+\chi_{i}^{\dagger} \sigma_{i}^{2}\right] \\
+ & h^{2} \sum_{i}\left|\chi_{i}\right|^{2}\left|\sigma_{i}\right|^{2}+\frac{g^{2}}{4} \sum_{i}\left|\chi_{i}\right|^{4}+\frac{h^{2}}{4} \sum_{i}\left|\sigma_{i}\right|^{4}, \\
-\mathcal{L}_{F}= & \frac{g}{2} \sum_{i}\left(\phi-M_{i}\right) \bar{\psi}_{\chi_{i}} P_{L} \psi_{\chi_{i}}+\frac{g}{2} \sum_{i}\left(\phi-M_{i}\right) \bar{\psi}_{\chi_{i}} P_{R} \psi_{\chi_{i}} \\
& +\frac{h}{2} \sum_{i} \chi_{i} \bar{\psi}_{\sigma_{i}} P_{L} \psi_{\sigma_{i}}+\frac{h}{2} \sum_{i} \chi_{i}^{\dagger} \bar{\psi}_{\sigma_{i}} P_{R} \psi_{\sigma_{i}} \\
& +h \sum_{i} \sigma_{i} \bar{\psi}_{\sigma_{i}} P_{L} \psi_{\chi_{i}}+h \sum_{i} \sigma_{i}^{\dagger} \bar{\psi}_{\sigma_{i}} P_{R} \psi_{\chi_{i}} .
\end{aligned}
$$

Note that the bare masses are $m_{\psi_{\chi_{i}}}=g\left(\phi-M_{i}\right)=m_{\chi_{i}}$ at zero temperature for unbroken SUSY. ${ }^{2}$ This is in agreement with [38] upon rescaling the couplings $g$ and $h$ in the superpotential by $1 / 2$ factors.

At finite temperature, both the $\chi_{i}$ and the $\psi_{\chi_{i}}$ receive thermal mass corrections. The contributions of the $\sigma_{i}$ and $\psi_{\sigma_{i}}$ fields to the latter have been computed in [38] and have been shown to be identical for both $\chi_{i}$ and $\psi_{\chi_{i}}$, corresponding to $h^{2} T^{2} / 8$ taking into account the coupling normalization differences. The $\chi_{i}$ scalars also receive thermal corrections from their self-interactions $g^{2}\left|\chi_{i}\right|^{4} / 4$. Noting that these interactions give a contribution to their tree-level mass $\partial^{2} V / \partial \chi_{i} \partial \chi_{i}^{\dagger}=g^{2}\left|\chi_{i}\right|^{2}$ and taking into account the contribution of the $\chi_{i}$ fields to the thermal effective potential

$$
\Delta V_{T} \subset 2 \times \frac{m_{\chi_{i}}^{2}}{24} T^{2}=\frac{g^{2}}{12} T^{2}\left|\chi_{i}\right|^{2}+\cdots,
$$

where we have taken into account the 2 d.o.f. for complex scalars, this yields a thermal mass correction $g^{2} T^{2} / 12$ to the $\chi_{i}$ fields. ${ }^{3}$ In summary, we obtain

\footnotetext{
${ }^{2}$ Note that in warm inflation SUSY is broken both by the finite temperature and the inflaton energy density. The latter should arise from an additional $\Phi$-dependent term in the superpotential that we have note included above and that will lead to a small splitting of the mass for the real and imaginary components of the $\chi_{i}$ scalar fields.

${ }^{3}$ The mass of the scalar $\sigma_{i}$ fields is also corrected by a similar factor (involving only the $h$ coupling) and, although the resulting masses are below the temperature, they will generically lie above $H$ in the parametric range relevant to our discussion. This implies that no scalar field other than the inflaton can sustain a slowly rolling background value that drives inflation.
}

$$
\Delta m_{\chi_{i}}^{2}=\frac{g^{2}}{12} T^{2}+\frac{h^{2}}{8} T^{2}, \quad \Delta m_{\psi_{\chi_{i}}}^{2}=\frac{h^{2}}{8} T^{2} .
$$

We also need to compute the finite temperature decay widths of the $\chi_{i}$ and $\psi_{\chi_{i}}$ fields. However, we only recall such calculations for Dirac fermions [39]; albeit the difference between Majorana and Dirac fermions is only in the overall factors of the decay width. Hence, we can first compute them at zero temperature to set the correct normalization factors in order to identify these global constants. In general, we have for the decay of a particle of mass $m$ at rest into a pair of massless particles

$$
\Gamma=\frac{S}{16 \pi m}|\mathcal{M}|^{2}
$$

where $S=1 / 2$ if the particles are identical and $S=1$ if they are distinct. The $\chi_{i}$ fields may decay via $\chi_{i} \rightarrow \sigma_{i} \sigma_{i}$ and $\chi_{i} \rightarrow \psi_{\sigma_{i}} \psi_{\sigma_{i}}$. In the first case, dropping the indices for simplicity, we may decompose the fields into their real and imaginary components via $\chi=\left(\chi_{R}+i \chi_{I}\right) / \sqrt{2}$ and analogously for $\sigma$. This yields the scalar interactions

$-\mathcal{L}_{\chi \sigma^{2}}=\frac{h g}{2 \sqrt{2}}\left(\phi-M_{i}\right)\left[\chi_{R} \sigma_{R}^{2}-\chi_{R} \sigma_{I}^{2}+2 \chi_{I} \sigma_{R} \sigma_{I}\right]$.

Hence, the $\chi_{R}$ scalar may decay into $\sigma_{R}$ or $\sigma_{I}$ pairs, while the $\chi_{I}$ scalar has only one decay channel $\chi_{I} \rightarrow \sigma_{R} \sigma_{I}$. For each of these decay channels, the vertex factor is $-i h g\left(\phi-M_{i}\right) /$ $\sqrt{2}$. Taking into account the $S=1 / 2$ factors in the $\chi_{R} \rightarrow$ $\sigma_{R} \sigma_{R}$ and $\chi_{R} \rightarrow \sigma_{I} \sigma_{I}$ channels, we then find that the decay widths are equal for both $\chi_{R}$ and $\chi_{I}$, being given by

$$
\Gamma_{\chi_{i}}^{S}=\frac{h^{2} g^{2}\left(\phi-M_{i}\right)^{2}}{32 \pi m_{\chi_{i}}} .
$$

The fermionic decay channel comes from the interaction term $\frac{1}{2} h \chi_{i} \bar{\psi}_{\sigma_{i}} \psi_{\sigma_{i}}$, where the vertex factor is simply $-i h$. Since the particles are identical in the final state and by computing the matrix element with the usual Feynman rules, this yields

$$
\Gamma_{\chi_{i}}^{F}=\frac{h^{2}}{32 \pi} m_{\chi_{i}}
$$

Noting that, at zero temperature, $m_{\chi_{i}}=g\left(\phi-M_{i}\right)$, we see that $\Gamma_{\chi_{i}}^{S}=\Gamma_{\chi_{i}}^{F}$ in this limit.

The fermions $\psi_{\chi_{i}}$ can decay as $\psi_{\chi_{i}} \rightarrow \sigma_{i} \psi_{\sigma_{i}}$ via the corresponding Yukawa term above, and this yields simply

$$
\Gamma_{\psi_{\chi_{i}}}=\frac{h^{2}}{16 \pi} m_{\psi_{\chi_{i}}} .
$$

Again, note that at zero temperature for unbroken SUSY we have $m_{\chi_{i}}=m_{\psi_{\chi_{i}}}=g\left(\phi-M_{i}\right)$, which yields identical 
total decay widths for the scalars and fermionic superpartners, as it should.

Once we have computed the decay widths at zero temperature, we identify each vertex factor squared from the decay of a scalar boson to fermions and the decay of a fermion to a scalar boson and a fermion. In the pole approximation (see Appendix A) we take the limit $T \rightarrow 0$ of the corresponding decay widths [41] and then by comparing them with Eqs. (20) and (21), we identify $g_{\chi_{i} \bar{\psi}_{\sigma_{i}} \psi_{\sigma_{i}}}^{2}=h^{2} / 4$ and $g_{\psi_{x_{i}} \sigma_{i} \psi_{\sigma_{i}}}^{2}=h^{2}$. From here we will use these overall factors for the rest of the computations.

\section{DISSIPATIVE INTERACTIONS}

The particle physics model considered in this work is inspired by string theory exhibiting $\mathcal{N}=1$ global supersymmetry, with the inflaton field coupled to massive modes of the string, as discussed in [42]. Several interactions are identified by the shifted couplings $g^{2}\left(\phi-M_{i}\right)^{2} \chi_{i}^{2}$ and $g\left(\phi-M_{i}\right) \bar{\psi}_{\chi_{i}} \psi_{\chi_{i}}$ for bosons $\chi_{i}$ and fermions $\psi_{\chi_{i}}$ respectively, with $\left\{M_{i}\right\}$ ranging over mass scales. This feature yields the name of distributed-mass model (DM model). In the subsequent segments we establish the relevant interplay the inflaton field has with the aforementioned fields. We will restrict to interactions such that the leading contribution will come dominantly from one-loop processes, when the decaying field is light. The key property of the DM model is that for a given temperature $T$, only the fields with masses $g^{2}\left(\phi-M_{i}\right)^{2} \lesssim T^{2}$ will contribute to the dissipation. Henceforth, we will refer to such a configuration of states as thermally excited sites. We will consider separately the dissipative processes associated with the excitation of the scalar $\chi_{i}$ fields, which may decay via $\chi_{i} \rightarrow \sigma_{i} \sigma_{i}$ or $\chi_{i} \rightarrow \bar{\psi}_{\sigma_{i}} \psi_{\sigma_{i}}$, and those associated with the excitation of the fermionic $\psi_{\chi_{i}}$ fields, which decay via $\psi_{\chi_{i}} \rightarrow \sigma_{i} \psi_{\sigma_{i}}$, with technical details of the computation given in Appendix A.

\section{A. Bosonic sector}

The dissipative coefficient arising from the pattern of interactions among the scalar component and the light states is given by $[8,10,11]$ (see also Appendix A)

$\Upsilon^{S}(\phi, T)=\sum_{i=1}^{\text {t.e. }} \frac{32 g^{4}}{\pi h^{2}\left[16 \frac{m_{\chi_{i}}^{2}}{\tilde{m}_{\chi_{i}}^{2}}+\frac{\tilde{m}_{\chi_{i}}^{2}}{T^{2}}\right]} \ln \left(\frac{2 T}{\tilde{m}_{\chi_{i}}}\right) \frac{\left(\phi-M_{i}\right)^{2}}{\tilde{m}_{\chi_{i}}}$,

where "t.e." means sum over all thermally excited sites. In the computation of the dissipative coefficient, the total decay rate for all processes is already taken into account, which is given by $[8,10,11]$ (see also Appendix A)

$$
\begin{aligned}
\Gamma_{\chi_{i}} & =\Gamma\left(\chi_{i} \rightarrow \sigma_{i} \sigma_{i}\right)+\Gamma\left(\chi_{i} \rightarrow \bar{\psi}_{\sigma_{i}} \psi_{\sigma_{i}}\right) \\
& =\frac{h^{2}}{128 \pi} \frac{T \tilde{m}_{\chi_{i}}}{\omega_{\chi_{i}}(p)}\left[16 \frac{m_{\chi_{i}}^{2}}{\tilde{m}_{\chi_{i}}^{2}}+\frac{\tilde{m}_{\chi i}^{2}}{T^{2}}\right],
\end{aligned}
$$

where $\omega_{\chi i}(p)=\sqrt{\tilde{m}_{\chi i}^{2}+|\mathbf{p}|^{2}}$ and $\tilde{m}_{\chi i}$ is the full $\chi_{i}$ mass, including the tree-level contribution $m_{\chi_{i}}$ and the thermal corrections computed above. We need to ensure that all light bosons remain in a nearly thermal state during inflation, so that the above result for the dissipation coefficient is a consistent approximation. For simplicity, we may take the thermal average of the above decay width, given by

$$
\bar{\Gamma}_{\chi_{i}}=\frac{1}{n_{B}} \int \frac{d^{3} p}{(2 \pi)^{3}} \Gamma_{\chi_{i}} f_{B},
$$

where $f_{B}(\mathbf{p} / T)$ is the Bose-Einstein distribution and $n_{B}\left(\tilde{m}_{\chi_{i}} / T\right)$ the associated number density. This yields in the limit $m_{\chi i} \lesssim T$ via a numerical approximation (see Appendix B)

$$
\bar{\Gamma}_{\chi_{i}} \simeq \frac{h^{2} f^{1 / 2}}{128 \pi}\left[\frac{16}{f} \frac{m_{\chi_{i}}^{2}}{T^{2}}+f\right] \frac{0.68}{\left(1+0.77 f^{1 / 2}\right)} T,
$$

where $f=f(g, h)=g^{2} / 12+h^{2} / 8$. Note that the above expression depends on the factor $m_{\chi_{i}} / T=g\left(\phi-M_{i}\right) / T$, so that different fields will decay at different rates. However, note that states for which $m_{\chi_{i}} / T=0$, corresponding to $\phi=M_{i}$, do not contribute to dissipation according to Eq. (22). Conversely, the heaviest states that can be thermally excited have $m_{\chi_{i}} \sim T$, and these are the ones that contribute the most to dissipation at any given time. Therefore, in analyzing the consistency of the model, namely whether the $\chi_{i}$ decay faster than expansion in order to remain close to thermal equilibrium, we will consider the states for which $m_{\chi_{i}} / T \simeq 1$.

\section{B. Fermionic sector}

The calculation of the dissipation coefficient can be done following essentially the same steps as in the "warm little inflaton" model [34-36], yielding

$\Upsilon^{F}(T)=\sum_{i=1}^{\text {t.e. }} C_{T}^{F} T, \quad C_{T}^{F} \simeq \frac{3 g^{2}}{h^{2}(1-0.34 \log (h))}$.

This calculation involves computing the finite temperature decay width of all interaction processes, which is given by

$$
\begin{aligned}
\Gamma_{\psi_{\chi_{i}}} & =\Gamma\left(\psi_{\chi_{i}} \rightarrow \sigma_{i} \psi_{\sigma_{i}}\right) \\
& =\frac{h^{2}}{16 \pi} \frac{T^{2} m_{\psi i}^{2}}{\omega_{\psi i}^{2}(p)|\mathbf{p}|}\left[F\left(\frac{k_{+}}{T}, \frac{\omega_{\psi i}(p)}{T}\right)-F\left(\frac{k_{-}}{T}, \frac{\omega_{\psi i}(p)}{T}\right)\right],
\end{aligned}
$$

where, neglecting the masses of the decay products $\sigma$ and $\psi_{\sigma}$, we have $\omega_{\psi i}(p)=\sqrt{\tilde{m}_{\psi i}^{2}+|\mathbf{p}|^{2}}, k_{ \pm}=\left(\omega_{\psi i}(p) \pm|\mathbf{p}|\right) / 2$, and 


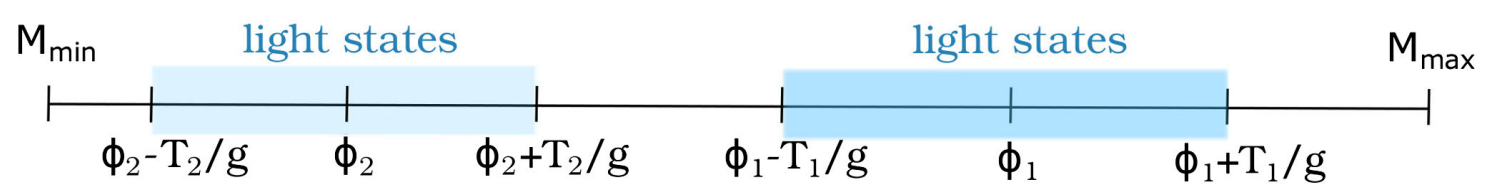

FIG. 1. Schematic representation of the time evolution in the model assuming that the temperature decreases.

$$
\begin{aligned}
F(x, y)= & x y-\frac{x^{2}}{2}+(y-x) \ln \left(\frac{1-e^{-x}}{1+e^{x-y}}\right) \\
& +\operatorname{Li}_{2}\left(e^{-x}\right)+\operatorname{Li}_{2}\left(-e^{x-y}\right),
\end{aligned}
$$

where $\mathrm{Li}_{2}(z)$ is the dilogarithm function. Note that the mass of the light fields is corrected by a factor of $h^{2} T^{2} / 8$ due to their interactions with the thermal bath fields $\psi_{\sigma_{i}}$ and $\sigma_{i}$, which we will take to be dominant over the inflaton contribution; i.e., we take $\tilde{m}_{\psi i} \simeq h^{2} T^{2} / 8$ in the above computation of the decay width. We need to ensure that all light fermions remain in a nearly thermal state during inflation, so that the above result for the dissipation coefficient is a consistent approximation. For simplicity, we may take the thermal average of the above decay width in the high-temperature and ultrarelativistic region:

$$
\bar{\Gamma}_{\psi_{\chi_{i}}}=\frac{1}{n_{F}} \int \frac{d^{3} p}{(2 \pi)^{3}} \Gamma_{\psi_{\chi_{i}}} f_{F}
$$

where $f_{F}(\mathbf{p} / T)$ is the Fermi-Dirac distribution and $n_{F}\left(\tilde{m}_{\chi_{i}} / T\right)$ the associated number density. This yields in the limit $m_{\psi_{\chi_{i}}} \lesssim T$

$$
\bar{\Gamma}_{\psi_{\chi_{i}}} \simeq 10^{-3}\left[1-0.875 \ln \left(\frac{h^{2}}{8}\right)\right] h^{4} T .
$$

\section{MASS DISTRIBUTION FUNCTION}

In the DM model the dissipative mechanism is well described by the interaction between the inflaton $\phi$ and the finite number of fermion and boson fields that are light at any given time as the scalar field scans the tower of states. DM models are distinguished by how the mass sites are distributed. Such an idea has a natural realization with string theory, whereby the inflaton is suggestive of an excited string zero mode, which then interacts with massive string levels. Such a construction of DM models from string theory was shown in [9]. String levels can be highly degenerate, and the distribution of mass states was suggested in [9] to emerge as a fine structure splitting of such levels. Thus the pattern of splitting a level will depend on the properties of the string state, ultimately governed by the underlying string vacuum. In this respect different string vacua should imply different distributions of mass states. A first principles determination of such distributions is difficult and minimally requires a detailed study of string theory case by case for each different possible vacua. However, we can adopt a phenomenological approach and look at various types of mass distributions to see what type of inflation they can lead to. This would be a minimum first step to test the viability of such models. In this section we develop some basic properties of the mass distribution function.

In general, the mass of states labeled by $i$ is as discussed in Sec. III, $m_{\chi_{i}}^{2}=m_{\psi_{\chi_{i}}}^{2}=g^{2}\left(\phi-M_{i}\right)^{2}$, and so is governed by the parameter $M_{i}$. It is the distribution of these mass sites $M_{i}$ that the underlying theory should determine, but here we treat them as phenomenological parameters and examine different types of distributions of $M_{i}$ over mass sites labeled by $i$. To understand the meaning of such mass sites, suppose the inflaton field $\phi$ sat in the middle of some mass sites. Thus for all mass sites $\phi-T / g<M_{i}<\phi+T / g$, the corresponding fields $\chi_{i}$ and $\psi_{\chi_{i}}$ would be thermally excited. As some terminology, we will call the region $\Delta \phi$, surrounding the inflaton $\phi$ that has thermally excited fields, a thermal interval. Some examples of this are shown in Fig. 1. The idea of warm inflation in such models is that the inflaton rolls through a region with many such mass sites, thus thermally exciting for some time a given field; then once $\phi$ is far enough away, that field again is no longer thermally excited. This implies, as the inflaton slow rolls during inflation, a thermal interval surrounding it moves with it.

With no underlying theory to dictate such mass distributions, we will simply consider one type of construction to make the idea more tangible. We will assume the mass sites can be written as

$$
M_{i}=\phi+i \Delta M(\phi, T, m, g)
$$

where $\Delta M$ is the mass gap in the tower, which may in general be a dynamical function of the inflaton's expectation value and the ambient temperature, as well as of some intrinsic mass scale $m$ and couplings such as $g$. For instance, in a theory with compact extra dimensions, as is the case of string/M-theory, the mass gap in the KaluzaKlein tower is inversely proportional to the size of the extra dimensions, generically set by the expectation value of one or more moduli fields. If such moduli interact with the inflaton and/or the thermal bath, their expectation value may become dynamical (although slowly evolving) during 
inflation, exhibiting a $\phi$ and/or $T$ dependence. ${ }^{4}$ With this motivation in mind, we will take an effective field theory approach and consider functional forms of the mass gap that lead to simple forms for the dissipative coefficient, analyzing their dynamical and observational consequences.

In the expression above $i$ is an integer ranging from mass sites closest to $\phi$ at $i=1$ up to a maximum mass site that is thermally excited determined by $i_{\max } \Delta M=T / g$. During the course of the observable range of inflation (around 50-60 e-folds), the inflaton will traverse some distance covering many thermal intervals $\Delta \phi$, and in general, one needs mass distributions where $\Delta M \ll \Delta \phi$, so that the inflaton crosses many mass sites in each thermal interval. One expects at least tens of mass sites per thermal interval and hundreds to thousands of thermal intervals crossed during the observable period of inflation. In other words, in each $e$-fold of inflation one expects tens or a few hundred mass sites to be crossed. Thus thousands of string states will be thermally excited for a brief period of time in the course of such a warm inflation period.

With this form of mass distribution function, one can then obtain expressions for the dissipative coefficient and the effective potential. For the dissipative coefficient the sum that is required is

$\sum_{i=1}^{i_{\max }}\left(\phi-M_{i}\right)^{2}=\sum_{i=1}^{i_{\max }} i^{2} \Delta M^{2} \approx i_{\max }^{3} \Delta M^{2} \simeq T^{3} /\left(g^{3} \Delta M\right)$.

For example, if we wanted $\Upsilon \sim \phi$, it means considering $\Delta M \sim T /\left(g^{3} \phi\right)$ and similarly for other forms of the dissipation function.

In the landscape context, the idea is that there are a huge number of different vacua, where some subset can produce a distributed mass model and some subset of those could have a distribution of mass sites that gives a dissipative coefficient that leads to observably consistent warm inflation. Since the possible vacua is so huge, it is simply seen as a statistical possibility that somewhere in this landscape a mass distribution of a particular type can be found. One would need to see this in the anthropic way that there could be inflation of many different forms occurring over the landscape, but some will occur in a way favorable to create a universe like ours. In this respect choosing a function $\Delta M$ boils down to finding the type of functions that can lead to a universe that looks like ours. If that could successfully be realized, as we will examine in the next section, then this model would have phenomenological viability. One could

\footnotetext{
${ }^{4}$ Note that the bare masses of the fields in the tower relevant for the dissipative process, $g M_{i} \sim g \phi$, are typically very large and that, at any given time, only a finite number of states become light due to the inflaton field reducing their mass. Therefore, this scenario does not require a low Kaluza-Klein/string scale to be implemented in the context of string/M-theory.
}

then explore as a much bigger step whether it is possible to find such particular types of DM models from string theory, although we will not be exploring that question here.

We can follow the above prescription in order to evaluate the effective finite temperature potential, where for the sake of simplicity we evaluate only the bare masses of the $\chi$ 's and $\psi_{\chi}$ 's fields. Hence both bosonic and fermionic sectors can be estimated using [43-46] [see Eqs. (C7) and (C1)]

$$
V_{T}^{\chi_{i}} \simeq \frac{T^{5}}{g \Delta M}\left\{-\frac{\pi^{2}}{45}+\frac{1}{12}+\frac{1}{6 \pi}-\frac{1}{32 \pi^{2}}\left[\ln \left(\frac{\mu^{2}}{T^{2}}\right)-c_{b}\right]\right\}
$$

$V_{T}^{\psi_{\chi_{i}}} \simeq \frac{T^{5}}{g \Delta M}\left\{-\frac{7 \pi^{2}}{360}+\frac{1}{24}+\frac{1}{32 \pi^{2}}\left[\ln \left(\frac{\mu^{2}}{T^{2}}\right)-c_{f}\right]\right\}$

hence if we select $\Delta M \sim T /\left(g^{3} \phi\right)$, the total effective finite temperature potential becomes

$V_{T}^{\chi_{i}}+V_{T}^{\psi_{\chi_{i}}} \simeq \frac{g^{2} T^{3}}{24} \phi\left[-\pi^{2}+3+\frac{4}{\pi}+\frac{3}{4 \pi^{2}}\left(c_{b}-c_{f}\right)\right]$.

Since the thermal corrections to the inflaton potential are, for this particular case, linear in the field, they contribute to the $\epsilon_{\text {eff }}$ parameter and need to be taken into account. However, in the scenarios with a quartic chaotic potential $V(\phi)=\lambda \phi^{4}$ that we analyze in more detail below, we typically find $T / \phi \sim 10^{-5}$ and $\lambda \sim 10^{-15}-10^{-14}$, such that these thermal corrections give a negligible contribution to both the effective potential and its first derivative for any value of the coupling $g$ in the perturbative regime. In addition, in the scenarios where the mass gap is field independent, thermal corrections to the inflaton potential do not modify the slow-roll parameters.

\section{RESULTS}

Let us now analyze the inflationary dynamics for a quartic scalar potential, $V(\phi)=\lambda \phi^{4}$, taking into account both scalar and fermionic contributions to the dissipation coefficient. In addition, different types of mass distribution functions will be used to lead to dissipative coefficients of various forms, namely $\mathrm{\Upsilon} \propto T^{2}, \Upsilon \propto T$, and $\mathrm{\Upsilon} \propto \phi$. Finite temperature corrections to the effective potential are computed for each case. These effects can be controlled, thus preventing thermal effects from generating large contributions to the inflaton mass that could reintroduce the $\eta$ problem.

\section{A. $\Upsilon \propto T^{2}$}

For a homogeneous distribution of states in the tower, with constant mass splitting $\Delta M$, as proposed by $[8,10,11]$, the resulting scalar coefficients grow with temperature as $\Upsilon \propto T^{2}$, with fermions contributing only about $20 \%$ to the 
total dissipation as first estimated in $[8,10,11]$. Because of the adiabatic condition $\bar{\Gamma}_{\chi}^{S} / H>1$, one requires large values of $T_{*} / H_{*}$; quantitatively we have that at least $T_{*} / H_{*} \gtrsim 150$, which in turn yields values of $Q_{*} \gtrsim 10$. Our results here are consistent with those reported in $[8,10,11]$. However, in the strong dissipation regime it is now understood that the primordial perturbations have a growing mode $[29,30,47,48]$. The consequences of this growing mode are to tilt the spectrum of perturbations toward the blue-tilted region, $n_{s}>1$, and so are inconsistent with the CMB data. Thus, the DM model with a homogeneous mass distribution resides outside the observational window provided by Planck data [1]. Although this particular mass distribution is not consistent with observations, the basic idea of the model shows appealing features, and one can explore other types of mass distributions as we will now do.

\section{B. $\Upsilon=\tilde{C}_{\phi} \phi$ and $V(\phi)=\lambda \phi^{4}$}

We want to study this model within the strong dissipative regime, since we expect that this model fits perfectly in it. This facilitates all calculations, so we will use standard analytical tools; i.e., we may implement the standard slowroll parameters $\epsilon_{\phi}=M_{P}^{2}\left(V_{, \phi} / V\right)^{2} / 2$ and $\eta_{\phi}=M_{P}^{2} V_{, \phi \phi} / V$. Also we introduce another slow-roll parameter to take into account the variation of $\Upsilon$,

$$
\beta=M_{P}^{2} \frac{\Upsilon_{, \phi} V_{, \phi}}{\Upsilon V},
$$

and the slow-roll conditions are now given by $\epsilon_{\phi}<Q$, $\left|\eta_{\phi}\right|<Q, \beta_{\phi}<Q$. We use the slow-roll equations, at $Q \gg 1$, in order to find a direct relation between $Q$ and $\phi$, yielding

$$
Q=\frac{\tilde{C}_{\phi}}{\sqrt{3 \lambda}} \frac{M_{P}}{\phi},
$$

and the slow-roll parameters are

$$
\epsilon_{\phi}=\frac{8 M_{P}^{2}}{\phi^{2}}, \quad \eta_{\phi}=\frac{12 M_{P}^{2}}{\phi^{2}}, \quad \beta_{\phi}=\frac{4 M_{P}^{2}}{\phi^{2}} ;
$$

so in the strong dissipative regime inflation ends at $\epsilon_{\phi} \simeq Q$, and this yields the inflaton value

$$
\phi_{\mathrm{end}}=\frac{8 \sqrt{3 \lambda}}{\tilde{C}_{\phi}} M_{P}
$$

The number of $e$-folds during inflation in the aforementioned regime is

$$
\begin{aligned}
N_{e} & =-\frac{1}{M_{P}^{2}} \int_{\phi_{*}}^{\phi_{\text {end }}} d \phi \frac{Q V}{V_{\phi}}=-\frac{\tilde{C}_{\phi}}{4 \sqrt{3 \lambda} M_{P}} \int_{\phi_{*}}^{\phi_{\text {end }}} d \phi \\
& =\frac{\tilde{C}_{\phi}}{4 \sqrt{3 \lambda}}\left[\left(\frac{\phi_{*}}{M_{P}}\right)-\left(\frac{\phi_{\text {end }}}{M_{P}}\right)\right] .
\end{aligned}
$$

Hence the inflaton value at the horizon crossing is

$$
\phi_{*}=\frac{4 \sqrt{3 \lambda}\left(N_{e}+2\right)}{\tilde{C}_{\phi}} M_{P}=2 \sqrt{\frac{N_{e}+2}{Q_{*}}} M_{P} .
$$

Once we have determined $\phi_{*}$, we can evaluate the observables at 50-60e-folds before inflation ends. Before we proceed, one should note that in the strong dissipative regime many parameters and formulas simplify; in fact, one of them is an analytic approximation to the density perturbation amplitude. Recall that for $T>H$, the dominant contributions to the primordial perturbation spectrum are thermal fluctuations of the inflaton field, as opposed to the conventional quantum fluctuations in cold inflation models. Upon exiting the horizon these thermal fluctuations freeze out as classical perturbations, and during slow roll at $Q \gg 1$ the amplitude of the curvature perturbation power spectrum is given by $[28,49]$

$$
\Delta_{\mathcal{R}}^{2} \simeq \frac{9}{4 \pi^{2}} \frac{H_{*}^{5} T_{*} Q_{*}^{5 / 2}}{V_{\phi_{*}}^{2}},
$$

where all quantities are evaluated at horizon crossing. Hence the scalar spectral index $n_{s}-1 \simeq d \ln \Delta_{\mathcal{R}}^{2} / d N_{e}$ is modified as well, becoming [28]

$$
\begin{aligned}
n_{s} & =1+\frac{3}{2}\left(\frac{\eta_{V}}{Q}-\frac{3}{2} \frac{\epsilon_{V}}{Q}-\frac{3}{2} \frac{\beta_{V}}{Q}\right)=1-\frac{9}{4}\left(\frac{2 \sqrt{3 \lambda}}{\tilde{C}_{\phi}} \frac{M_{P}}{\phi_{*}}\right) \\
& =1-\frac{9}{4}\left(\frac{1}{N_{e}+2}\right) .
\end{aligned}
$$

Remarkably, note that $n_{s}$ depend only on the number of $e$-folds: $n_{s}\left(N_{e}=50\right)=0.9567$ and $n_{s}\left(N_{e}=60\right)=0.9637$, where at $60 e$-folds this scalar spectral index agrees outstandingly with Planck data [1]. The tensor-to-scalar ratio $r=\Delta_{t}^{2} / \Delta_{\mathcal{R}}^{2}$, as mentioned above, is typically reduced by the modifications to the scalar curvature perturbations introduced due to dissipation, which is basically a function of $Q_{*}$. We illustrate this fact by using the slow-roll dynamics, where the ratio $r$ can be written as

$r=\frac{2 H_{*}^{2}}{\pi^{2} M_{P}^{2} \Delta_{\mathcal{R}}^{2}}=\frac{2 \lambda}{3 \pi^{2} \Delta_{\mathcal{R}}^{2}} \frac{\phi_{*}^{4}}{M_{P}^{4}}=\frac{32 \lambda}{3 \pi^{2} \Delta_{\mathcal{R}}^{2}} \frac{\left(N_{e}+2\right)^{2}}{Q^{2}}$.

The value of $\lambda$ is fixed by using the normalization of the amplitude of the primordial spectrum $\Delta_{R}^{2} \simeq 2.2 \times 10^{-9}$ [1]. Using the slow-roll equations in Eq. (42), we have

$$
\lambda=\left(\Delta_{\mathcal{R}}^{2}\right)^{4 / 3}\left(\frac{\left(12 \pi^{4} C_{R}^{1 / 2}\right)^{2 / 3}}{\left(N_{e}+2\right)^{3}}\right)
$$

where $C_{R}=\pi^{2} g_{\text {eff }} / 30$, and $g_{\text {eff }}=1+15 N_{M} / 4, N_{M}$ being the no of bosonic $\chi_{i}$ (fermionic $\psi_{i}$ ) light d.o.f. at horizon crossing. The tensor-to-scalar ratio is then just a function of $Q_{*}$ and $N_{e}$, 


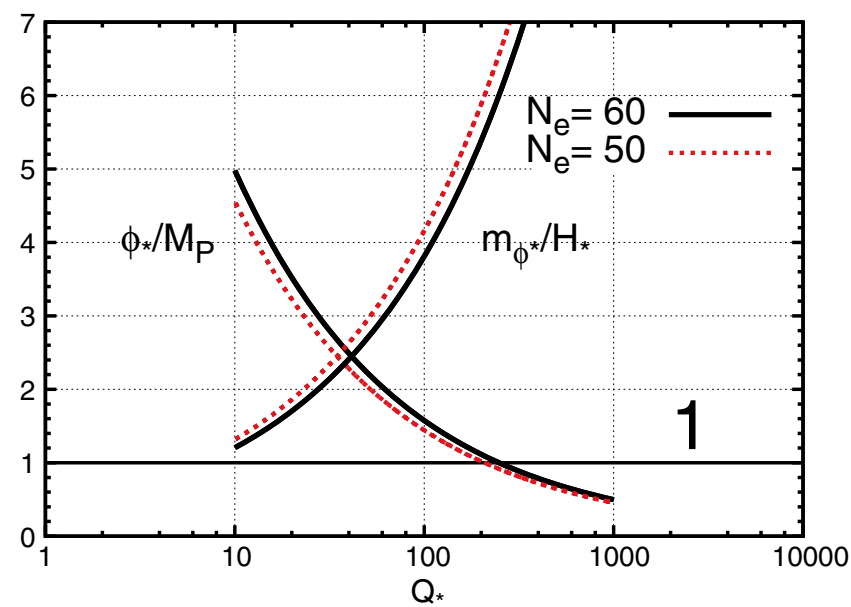

FIG. 2. Ratios $m_{\phi_{*}} / H_{*}$ and $\phi_{*} / M_{P}$, as a function of the dissipative ratio $Q_{*}$, within a DM scenario with a dissipative coefficient of the form $\Upsilon \propto \phi$ described by a quartic potential for 50 (red dashed line) and 60 (black solid line) $e$-folds of inflation. Note that $m_{\phi_{*}} / H_{*}$ is always larger than 1 for $Q_{*}>10$, while $\phi_{*} / M_{P}<1$ (sub-Planckian) at $Q_{*} \sim 210$.

$$
r=\frac{64\left(6 \Delta_{\mathcal{R}}^{2}\right)^{1 / 3}}{3 Q_{*}^{2}\left(N_{e}+2\right)}
$$

As one expected, the tensor-to-scalar ratio is highly suppressed by dissipation. In fact, this ratio lies in the region $10^{-9}<r \lesssim 10^{-4}$ at $10 \leq Q_{*} \leq 1000$, for $N_{M}=O(10-100)$ ), having the bigger suppression at the largest $Q_{*}$.

We can also look at the ratios $\phi_{*} / M_{P}$ and $m_{\phi_{*}} / H_{*}$ in order to illustrate that for this model the relevant scales can indeed happen in the sub-Planckian region, as shown in Fig. 2. This occurs thanks to the high dissipation dynamics,

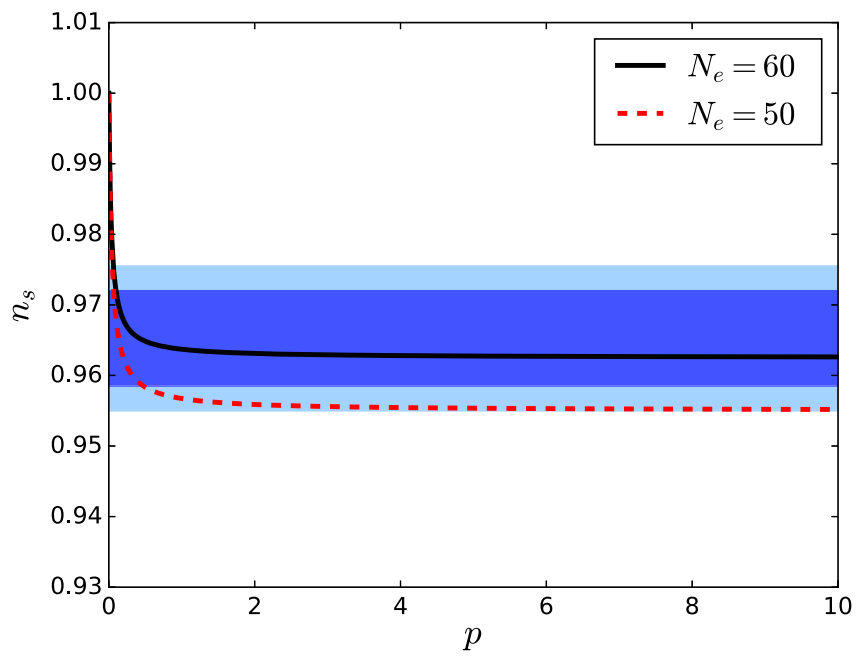

since in the WI field potentials that are not flat enough to allow the standard slow-roll inflaton evolution (i.e., they do not have large enough initial inflaton values so not "sufficient" inflation takes place) can, in fact, lead to a longer period of inflation due to the extra friction induced by $\Upsilon$. Hence, for small (even sub-Planckian) $\phi_{*}$ inflation can last 50-60 $e$-folds in the strong dissipative regime.

We can even generalize the above results for a dissipative coefficient of the form $\Upsilon=C_{\phi} \phi^{p} m^{1-p}$, where $m$ is a mass scale and $p$ is a free parameter. For this generic case, the scalar spectral index and the tensor-to-scalar ratio become very simple expressions that yield a remarkable agreement with Planck legacy [1] (see Fig. 3):

$n_{s}=1-\frac{9}{4}\left(\frac{p}{p N_{e}+2}\right), \quad r=\frac{32 \lambda}{3 \pi^{2} \Delta_{\mathcal{R}}^{2}} \frac{\left(p N_{e}+2\right)^{2}}{Q^{2}}$.

The good agreement between WI with a dissipative coefficient of the form $\Upsilon=C_{\phi} \phi^{p} m^{1-p}$ described by a chaotic quartic potential has been reported before. For instance, the authors of [30] analyzed the $\Upsilon \propto \phi^{2}$ case, finding an observably favorable region for $Q \gg 1$. On the other hand, the tensor-to-scalar ratio is enhanced by larger $p$, so for very large $p$ this ratio $r$ can indeed become bigger than 1 . The limits on ongoing and planned $B$-mode polarization experiments [50] suggest sensitivity down to $r \gtrsim 10^{-3}$. This leaves open the door to access desirable values of $r$ for very large $Q_{*}$.

\section{C. $\Upsilon=C_{T} T$ and $V(\phi)=\lambda \phi^{4}$}

We can ensure a linear dissipative coefficient by considering a DM model with a constant number of bosonic

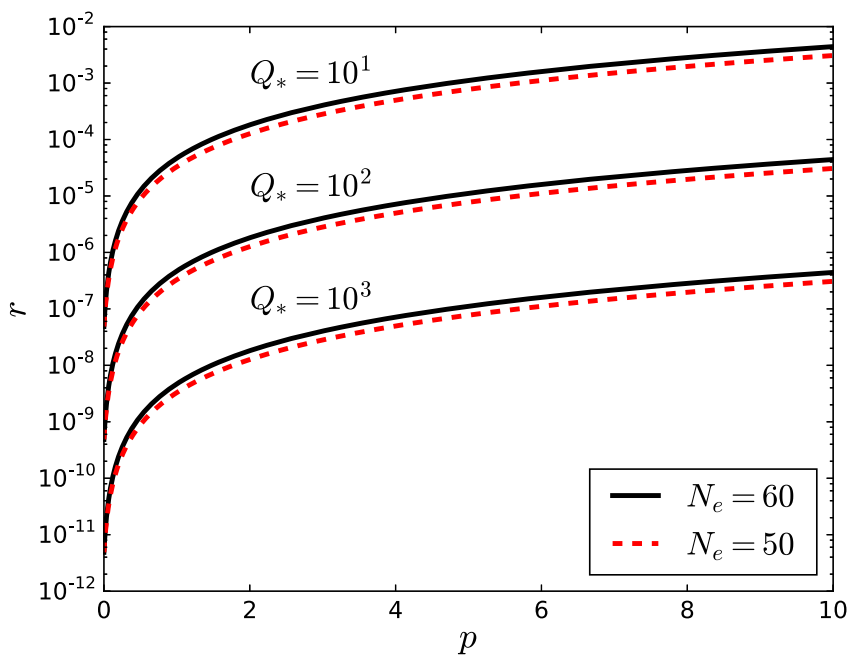

FIG. 3. Observational predictions of the DM scenario with a dissipative coefficient of the form $\Upsilon \propto \phi^{p}$ described by a quartic potential for 50 (red dashed line) and 60 (black solid line) $e$-folds of inflation. The plot on the left shows the spectral index $n_{s}$ as a function of the parameter $p$, while the plot on the right shows the tensor-to-scalar ratio $r$ as a function of $p$ for three values of $Q_{*}$. The blue contours correspond to the $68 \%$ and 95\% C.L. results from Planck 2018 TT, TE, EE + lowE + lensing data [1]. Note that $n_{s}$ lies well inside the Planck contours at $p \geq 1$. On the other hand, $r$ gets enhanced for larger $p$. 

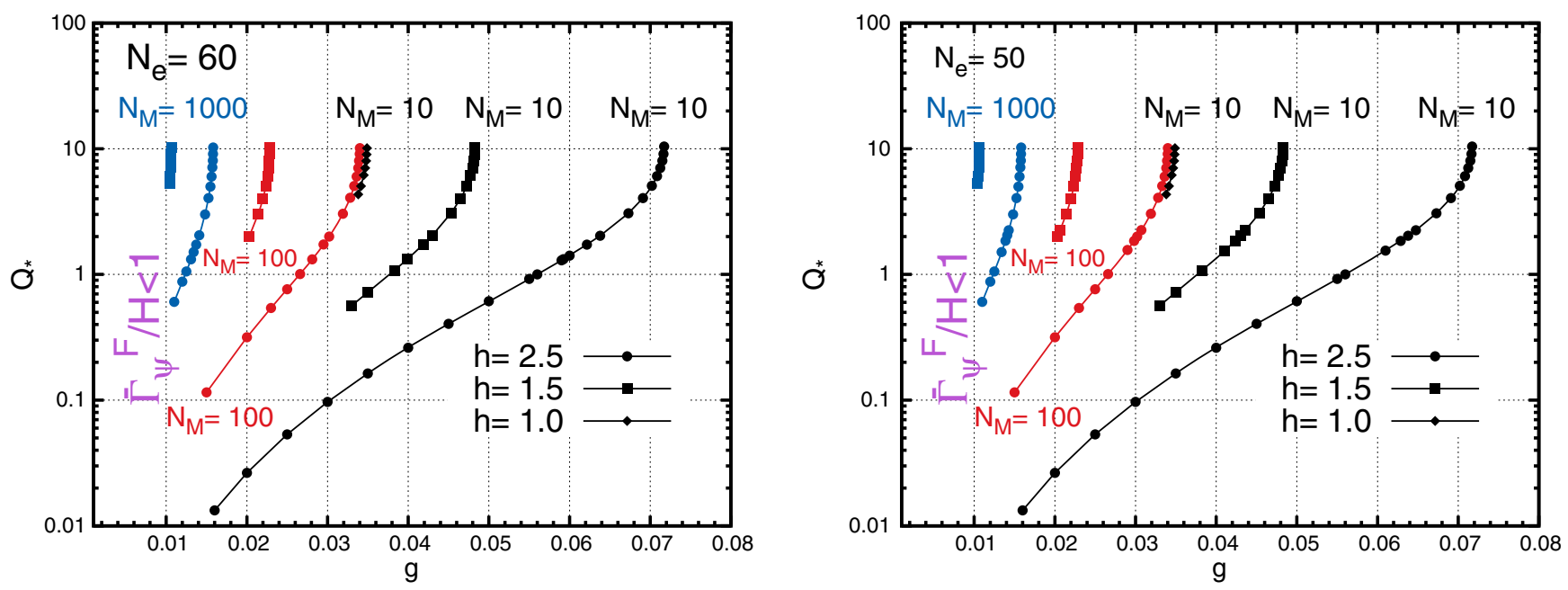

FIG. 4. Dissipative ratio at the horizon crossing, $Q_{*}$, as a function of the coupling $g$, within a DM scenario with a dissipative coefficient of the form $\Upsilon \propto T$ described by a quartic potential and $N_{e}=50$ (right) or $N_{e}=60$ (left) $e$-folds of inflation. We consider different numbers of light states $N_{M}=10,100$, and 1000, shown by the black, red, and blue curves, respectively, as well as different values of the Yukawa coupling $h=2.5$ (circles), $h=1.5$ (rectangles), and $h=1.0$ (diamonds). Note that the adiabatic conditions $\bar{\Gamma}_{\chi_{i}} / H \gtrsim 1$ are not satisfied for small values of $g$.

(fermionic) light d.o.f. $N_{M}$ during the evolution of the inflaton field. This is equivalent to having in the continuum limit a density of states that goes inversely with $T$, such that $N_{M} \simeq 2 \operatorname{Tn}(\phi) / g$. Using this, and performing the sums in Eqs. (22) and (26) in the continuum limit, we then have

$$
\begin{aligned}
\Upsilon^{S}(\phi, T) & =N_{M} C_{T}^{S} T, \\
C_{T}^{S}=\frac{2 g^{2} \sqrt{f}}{\pi} \ln \left(\frac{2}{\sqrt{f}}\right)\left[1-\frac{f}{4} \operatorname{ArcTan}\left(\frac{4}{f}\right)\right], & \\
\Upsilon^{F}(\phi, T) & =N_{M} C_{T}^{F} T,
\end{aligned}
$$

where $C_{T}^{F}$ was given in Eq. (26). The observational predictions will depend now on the couplings $g$ and $h$, and the parameter $N_{M}$.

To ensure the consistency of the analysis, namely the computation of the dissipation coefficients, we must verify that both the bosonic and the fermionic fields coupled to the inflaton are kept close to thermal equilibrium, thus requiring $\bar{\Gamma}_{\chi_{i}}, \bar{\Gamma}_{\psi_{\chi_{i}}}>H$. Since both average decay widths are proportional to the temperature and smaller than the latter for perturbative couplings, if the latter conditions are satisfied, we also ensure that $T \gtrsim H$ throughout inflation, thus validating the flat space approximation used in computing the dissipative coefficients. For the quartic potential, the ratio $T / H$ increases during inflation, being proportional to the dissipative ratio $Q$, such that we only have to ensure that these conditions are met at the moment of horizon crossing for the relevant CMB scales.

We note that in another realization of warm inflation with light fields, the Warm Little Inflaton (WLI) scenario, one has to impose additional constraints on the temperature during warm inflation so as to keep it above the mass of the fermionic fields coupled to the inflaton and below the underlying symmetry breaking scale. Such conditions are absent in the DM model, which is therefore less constraining. This comes, of course, at the expense of considering a whole tower of fields coupled to the inflaton, although only a small number of fields contributes effectively to dissipation at any given time.

In our analysis, we have also included the one loop thermal effective potential, computed in Appendix C. The bosonic contribution is given in Eq. (C9), while the fermionic one is given in Eq. (C6). From those expressions, one can see that the contribution of the finite temperature corrections to the derivatives of the effective potential is absent in this model. We have kept anyway the subdominant thermal contribution to the potential, setting the renormalization scale such as $\mu=\exp \left(c_{f} / 2\right) T_{*}$, where $T_{*}$ is the temperature at the horizon crossing. It is worth mentioning that by choosing the renormalization scale through another prescription, for instance, $\mu=\exp \left(c_{b} / 2\right) T *$, the outcome is not substantially altered.

With a $T$-dependent dissipative coefficient, the amplitude of the primordial spectrum Eq. (8) includes the growing mode function $G\left(Q_{*}\right)$. For a chaotic quartic potential, it was obtained numerically in $[34,36]$

$$
G\left(Q_{*}\right) \simeq 1+0.0185 Q_{*}^{2.315}+0.335 Q_{*}^{1.364},
$$

which alongside the dynamical evolution equations described in the previous section allows us to compute the inflationary observables for the chaotic model for $50-60 e$-folds of inflation, provided that the adiabatic conditions discussed above are satisfied. The inflaton 

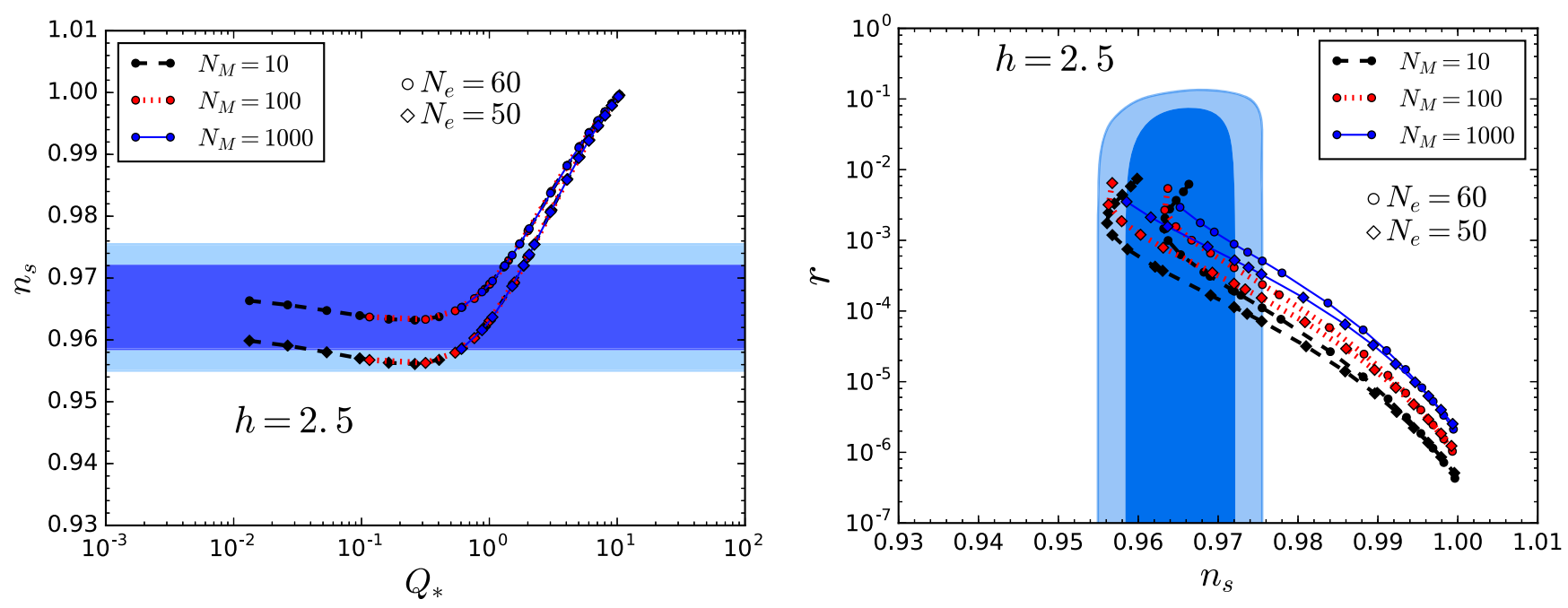

FIG. 5. Observational predictions of the DM scenario with a dissipative coefficient of the form $\Upsilon \propto T$ described by a quartic potential for 50 (diamonds) and 60 (circles) $e$-folds of inflation, a Yukawa coupling $h=2.5$, and three different values of the number of light states $N_{M}=10,100,100$ in colors (line style) black (dashed line), red (pointed line), and blue (solid line), respectively. The plot on the left shows the spectral index $n_{s}$ as a function of the dissipative ratio at the horizon crossing, $Q_{*}$, while the plot on the right shows the allowed trajectories in the $\left(n_{s}, r\right)$ plane. The blue contours correspond to the $68 \%$ and $95 \%$ C.L. results from Planck 2018 $\mathrm{TT}, \mathrm{TE}, \mathrm{EE}+$ lowE + lensing data [1].

self-coupling $\lambda$ is fixed as before by using the observed amplitude of curvature perturbations, $\Delta_{R}^{2} \simeq 2.2 \times 10^{-9}[1]$, at 50-60e-folds before the end of inflation.

For the quartic potential, we show in Fig. 4 the obtained values of the dissipative ratio at the horizon crossing, $Q_{*}$, as a function of the coupling $g$, for different numbers of light states and values of the Yukawa coupling $h$. We note in this figure that the adiabatic condition $\bar{\Gamma}_{\chi_{i}} / H \gtrsim 1$ for the fermions is only satisfied for $g \gtrsim 0.01$. We also find a lower bound $Q_{*}>0.01$ for the smallest number of fields considered, and the adiabatic conditions imply generically $h \gtrsim 1$. We only show in this figure values of $Q_{*}<11$ since, as we will see below, larger values are incompatible with observational data [1].

This behavior had been reported before for the WLI model [34-36]. For instance, the analysis in [36] presented a rather similar lower bound on $g \gtrsim 0.01$ and $Q * \gtrsim 0.001$ due to the adiabatic condition, while the conditions on the temperature limit this coupling from above, $g \lesssim 0.1$, such that $Q * \lesssim 0.25$. However, the DM model yields a substantially wider consistent parametric range, particularly for the value of the dissipative ratio at the horizon crossing.

Note that for larger values of the number of light states $N_{M}$ and of the Yukawa coupling $h$ we obtain a much narrower range of consistent values for the coupling $g$, and the lower bound on $Q_{*}$ also increases with $N_{M}$ and $h$. We thus find scenarios where inflation can start in either the weak or the strong dissipation regimes, noting that $Q$ grows during inflation such that for a wide range of parameters one reaches $Q>1$ before the end of inflation, a necessary condition for radiation to dominate after the slow-roll regime with no further reheating [see Eq. (6)].
In Figs. 5-7 we show the predictions for the scalar spectral index and tensor-to-scalar ratio in the allowed parametric ranges, for different values of $h$ and $N_{M}$, exhibiting a remarkable consistency with the Planck legacy results. This is particularly relevant given that the quartic potential is already excluded by such a survey within the CI paradigm.

We find agreement with the Planck legacy data in the parametric ranges yielding values of $Q_{*} \lesssim 1$, which is easier to achieve for larger values of the Yukawa coupling, as well as a smaller number of light fields. For instance, we cannot find consistency with the Planck data for $h=1$, since smaller values of the Yukawa coupling lead to stronger dissipation at the horizon crossing. These results were expected, since the growing mode in the spectrum makes it more blue tilted with increasing $Q_{*}$.

In general, the tensor-to-scalar ratio lies in the range $10^{-4} \lesssim r \lesssim 10^{-2}$, for values of the scalar spectral index within the Planck window, being more suppressed for larger values of $Q_{*}$ (smaller Yukawa couplings and larger number of light fields $N_{M}$ ). This feature is quite generic, since a similar notable trend was obtained in [25,32-36]. Additionally, this significant agreement with Planck data is easily achieved with only a small number of light states $N_{M}$.

The remarkable agreement between the DM quartic model and the Planck data is similar to what had been obtained for the WLI quartic model [34-36], where the dissipation coefficient is also proportional to the temperature. However, while the more constraining conditions of the WLI model limit the consistent parametric range to $Q_{*} \lesssim 0.25$, we have found consistent DM scenarios with up to $Q_{*} \sim 1.85-2.25(1.32-1.73)$ for 50 (60) $e$-folds of inflation within the contours corresponding to the $68 \%$ 

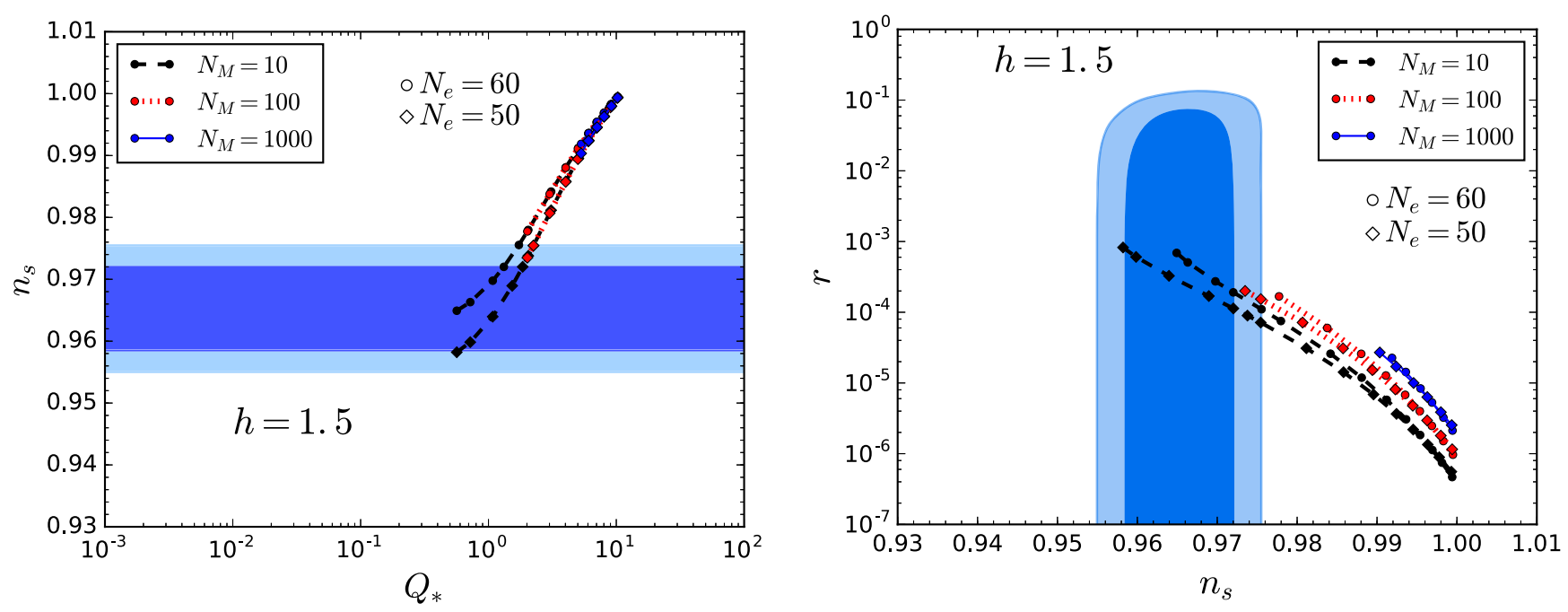

FIG. 6. Observational predictions of the DM scenario with a dissipative coefficient of the form $\Upsilon \propto T$ described by a quartic potential for 50 (diamonds) and 60 (circles) $e$-folds of inflation, a Yukawa coupling $h=1.5$, and three different values of the number of light states $N_{M}=10,100,100$ in colors (line style) black (dashed line), red (pointed line), and blue (solid line), respectively. The plot on the left shows the spectral index $n_{s}$ as a function of the dissipative ratio at the horizon crossing, $Q_{*}$, while the plot on the right shows the allowed trajectories in the $\left(n_{s}, r\right)$ plane. The blue contours correspond to the $68 \%$ and $95 \%$ C.L. results from Planck 2018 TT, TE, $\mathrm{EE}+$ lowE + lensing data [1].
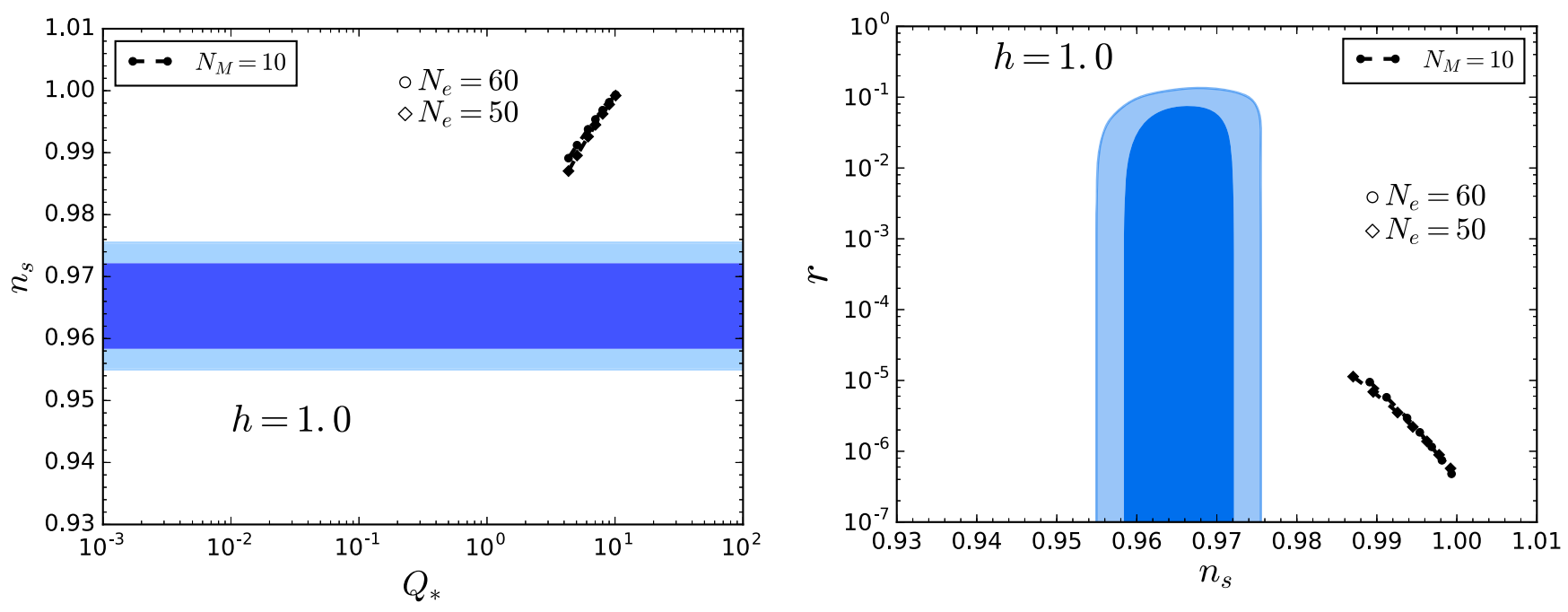

FIG. 7. Observational predictions of the DM scenario with a dissipative coefficient of the form $\Upsilon \propto T$ described by a quartic potential for 50 (diamonds) and 60 (circles) $e$-folds of inflation, a Yukawa coupling $h=2.5$, and three different values of the number of light states $N_{M}=10,100,100$ in colors (line style) black (dashed line), red (pointed line), and blue (solid line), respectively. The plot on the left shows the spectral index $n_{s}$ as a function of the dissipative ratio at the horizon crossing, $Q_{*}$, while the plot on the right shows the allowed trajectories in the $\left(n_{s}, r\right)$ plane. The blue contours correspond to the $68 \%$ and $95 \%$ C.L. results from Planck 2018 TT, TE, EE + lowE + lensing data [1].

and $95 \%$ C.L. results from Planck 2018 TT, TE, $\mathrm{EE}+$ lowE + lensing data [1]. In addition, this noteworthy consistency with Planck, which also leads to a finite range for the tensor-to-scalar ratio, might be relevant in the search of primordial gravitational waves via $B$-mode polarization experiments in the near future (see, e.g., [50]).

Finally, we evaluate both swampland criteria within the observational window provided by Planck [1], and we find that none is fully satisfied, with $10 \lesssim|\Delta \phi| / M_{P} \lesssim 21$ and $0.19 \lesssim M_{P}\left|V_{\text {eff }, \phi}\right| / V_{\text {eff }} \lesssim 0.4$ for $60 e$-folds and $7 \lesssim$ $|\Delta \phi| / M_{P} \lesssim 19$ and $0.2 \lesssim M_{P}\left|V_{\text {eff }, \phi}\right| / V_{\text {eff }} \lesssim 0.55$ for 50 $e$-folds. Nevertheless, the scenarios for which $Q_{*} \sim 1$ are closer to satisfying these criteria than the corresponding cold inflation scenarios could, in fact, satisfy them if they were somewhat relaxed. For instance, it has been discussed in $[18,51]$ that $c$ could be as small as $\mathcal{O}\left(10^{-11}\right)$, and it does 
not object, perceiving de Sitter vacua in string landscapes. Moreover, there has been some controversy on the precise values for $\Delta$ and $c$, given that they depend on the specific string model being considered [52]. Basically, if $\Delta$ were increased by an order of magnitude and $c$ were decreased by around $c \sim 0.4$, both swampland criteria could easily be fulfilled for $Q_{*} \gtrsim 1$.

\section{CONCLUSIONS}

Warm inflation offers a unique description of the early universe cosmology. It is a picture of an inflationary dynamics in which the state of the universe during inflation is not the vacuum state, but rather an excited statistical thermal state. It introduces dissipation into the inflationary dynamics, which can be well explained by first principles of a quantum multifield theory. Besides, this approach has several attractive features. For instance, the additional friction may ease the required flatness of the inflaton potential alleviating the swampland criterion. Also, even if radiation is subdominant during inflation, it may smoothly become the leading component if $Q \gtrsim 1$ at the end of inflation $\left(\epsilon_{\text {eff }} \sim 1+Q\right)$, with no need for a separate reheating period. ${ }^{5}$ It also may explain the nature of the classical inhomogeneities observed in the CMB, since for WI the fluctuations of the inflaton are thermally induced; hence there is no need to explain the troublesome quantum-toclassical transition problem of CI, due to the purely quantum origin of the CI density perturbations. Furthermore, it was shown early on [10], in fact, in studying the DM model, that the presence of dissipation and temperature lowers the energy scale of inflation in monomial models such as $\lambda \phi^{4}$ in comparison to the cold inflation results. This was further developed in $[25,32,33]$, with the prediction for a low tensorto-scalar ratio, which subsequently has been shown to be consistent with data [1]. These observational predictions for warm inflation were most recently tested in [54] with good agreement with Planck data.

The ultimate goal in building inflation models is to have them consistent with observational data and also be theoretically consistent. The latter has many levels of criteria. In [55], based on general properties of quantum field theory, conditions were listed for theoretically consistent warm inflation models. In fact, a subset of those conditions would also apply to cold inflation models. Amongst the criteria stated were that $\phi<M_{P}$ and $m_{\phi}>H$. The former was based on general consideration

\footnotetext{
${ }^{5}$ Although we have not specified how the fields in the tower interact with the Standard Model d.o.f., which is a modeldependent issue, these will generically be excited at the end of inflation once the Hubble rate is sufficiently reduced, given the large values of the temperature $T \sim 10^{12-13} \mathrm{GeV}$ when radiation becomes dominant. In fact, it would require extremely small couplings between the tower and Standard Model states to prevent the latter's thermalization before nucleosynthesis at $T \sim$ $10 \mathrm{MeV}$ (see also [53]).
}

of low-energy effective field theory. The latter is so that inflaton particles remained sub-Hubble scale and is basically the $\eta$ condition. However, in contrast to cold inflation, where this condition is something one needs to build the model around, in warm inflation due to the presence of dissipation this condition can hold and inflation can still occur. In Sec. VI explicit examples of warm inflation models are shown where $\phi<M_{p}$ and $m_{\phi}>H$, such as in Fig. 2. These are very stringent requirements for an inflation model, and no cold inflation models can achieve both of them. The swampland criteria are basically contained within these criteria. In this paper we have found warm inflation models that are consistent with all the criteria stated in [55] and so in turn are also consistent with the swampland criteria. Supergravity corrections will also, in principle, be under control in such supersymmetric scenarios with sub-Planckian field values, although scenarios with $\phi>M_{p}$ may also be viable in supergravity with noncanonical choices of the Kähler potential (see, e.g., [56]).

The distributed mass model of warm inflation studied in this paper develops the original studies to include different field- and temperature-dependent mass distributions and makes accurate comparisons with the most recent Planck data. This model fits naturally with the string landscape picture, with different mass distributions in the model ultimately arising from different possible string vacua. We found, for example, for the $\Upsilon \sim \phi$ model with the $\lambda \phi^{4}$ potential, full consistency with the conditions in [55] and therefore also consistency with the swampland criteria. As the $\lambda \phi^{4}$ potential in many respects is the benchmark inflation model, this consistency is a very promising result for both warm inflation and the DM implementation of landscape phenomenology. We also examined other types of mass distribution functions with varied levels of success. Overall this shows these models have a robust range of possibility. These results encourage further understanding of DM models in particular in the context of string theory building on the ideas developed in [9].

If one accepts string theory and thus its landscape property as the fundamental description of nature, then it forces a rethink on the relevance of simplicity for an inflation model. Any point on the landscape that leads to a consistent effective field theory model which agrees with observational data is just as good as any other. We refrain from calling this what it is, but that is the path this line of reasoning evidently takes us down. From the present vantage point with so many possible vacua, it would seem easy to argue that any working low-energy effective model leading to observationally consistent inflation can be found somewhere in this vast landscape. Alternatively said, it is hard to argue that simple low-energy models are the only ones that could arise from this landscape. It is possible that future work in string theory will reduce the number of viable vacua, but given the starting point, that seems a difficult task. From another viewpoint, the loss of predictivity that comes with the landscape property, equally encourages objective 
thinking whether string theory is the best way to approach the ultraviolet completion problem.

In a certain way of looking at it, DM models are rather complicated in that there are many fields and several conditions to examine and calculate. However, from another perspective they are also simple in that they are renormalizable models with canonical kinetic terms and require no assumptions of the coupling of gravity. Because of these properties, they are very amenable to being part of an extension of the Standard Model. All said, from the landscape perspective, DM models are viable models that are interesting to further explore.

\section{ACKNOWLEDGMENTS}

R. H.-J. acknowledges CONACyT for financial support. A. B. is supported by STFC. M. B.-G. is partially supported by MINECO Grant No. FIS2016-7819-P and Junta de Andalucía Project No. FQM-101. J.G. R. is supported by the FCT Investigator Grant No. IF/01597/2015, partially by the H2020-MSCA-RISE-2015 Grant No. StronGrHEP-690904, and by the Centro de Investigação e Desenvolvimento em Matemática e Aplicações Project No. UID/MAT/04106/2019.

\section{APPENDIX A: DISSIPATIVE COEFFICIENT IN THE HIGH TEMPERATURE REGIME: POLE APPROXIMATION}

The leading contribution to the dissipative coefficient from the scalar $\chi_{i}$ fields has the following form $[39,40]$ :
$\Upsilon=\sum_{i=1}^{N_{M}} \frac{4}{T}\left(\frac{g^{2}}{2}\right)^{2}\left(\phi-M_{i}\right)^{2} \int \frac{d^{4} p}{(2 \pi)^{4}} \rho_{\chi}^{2} n_{B}\left(1+n_{B}\right)$,

where $n_{B}=\left[e^{p_{0} / T}-1\right]^{-1}$ is the Bose-Einstein distribution and $\rho_{\chi}$ is the spectral function for the $\chi_{i}$ field. The spectral function for the $\chi_{i}$ field entering in Eq. (A1) corresponds to the fully dressed propagator, including the effect of its finite decay width into $\sigma_{i}$ and $\psi_{\sigma_{i}}$ particles,

$$
\rho_{\chi}\left(p, p_{0}\right)=\frac{4 \omega_{p} \Gamma_{\chi_{i}}}{\left(p_{0}^{2}-\omega_{p}^{2}\right)^{2}+4 \omega_{p}^{2} \Gamma_{\chi_{i}}^{2}},
$$

where $\omega_{p}=\sqrt{\tilde{m}_{\chi i}^{2}+p^{2}}$ and $\tilde{m}_{\chi i}^{2}=m_{\chi i}^{2}+g^{2} / 12+h^{2} T^{2} / 8$. The leading contribution to the decay width of the $\chi_{i}$ fields is then the two-body decay $\chi_{i} \rightarrow \sigma_{i} \sigma_{i}$ and $\chi_{i} \rightarrow \bar{\psi}_{\sigma_{i}} \psi_{\sigma_{i}}$, where at finite temperature we include contributions from both decays and inverse decays, as well as thermal scattering off particles in the radiation bath. The first decay process has been computed in $[39,40]$ from the imaginary part of the $\chi_{i}$ self-energy at one-loop order, yielding

$$
\Gamma_{\chi_{i}}\left(\chi_{i} \rightarrow \sigma_{i} \sigma_{i}\right)=\frac{h^{2}}{32 \pi} \frac{m_{\chi i}^{2}}{\omega_{p}} F_{T}^{\sigma_{i} \sigma_{i}}\left(p, p_{0}\right)
$$

where

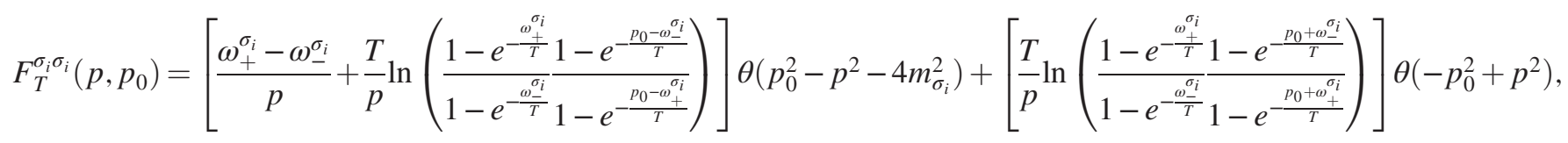

with $m_{\sigma_{i}}$ denoting the scalar $\sigma_{i}$ mass, $\theta(x)$ the Heaviside function, and

$$
\omega_{ \pm}^{\sigma_{i}}=\sqrt{\left(k_{ \pm}^{\sigma_{i}}\right)^{2}+m_{\sigma_{i}}^{2}}, \quad k_{ \pm}^{\sigma_{i}}=\frac{1}{2}\left|p \pm p_{0}\left(1-\frac{4 m_{\sigma_{i}}^{2}}{p_{0}^{2}-p^{2}}\right)^{1 / 2}\right|
$$

On the other hand, the leading contribution to the decay width of the $\chi_{i}$ fields into two fermions $\bar{\psi}_{\sigma_{i}} \psi_{\sigma_{i}}$ has been computed in [39] from the imaginary part of the $\chi_{i}$ self-energy at one-loop order, yielding

$$
\Gamma_{\chi_{i}}\left(\chi_{i} \rightarrow \bar{\psi}_{\sigma_{i}} \psi_{\sigma_{i}}\right)=\frac{h^{2}}{32 \pi \omega_{p}} F_{T}^{\bar{\psi}_{\sigma_{i}} \psi_{\sigma_{i}}}\left(p, p_{0}\right)
$$

where

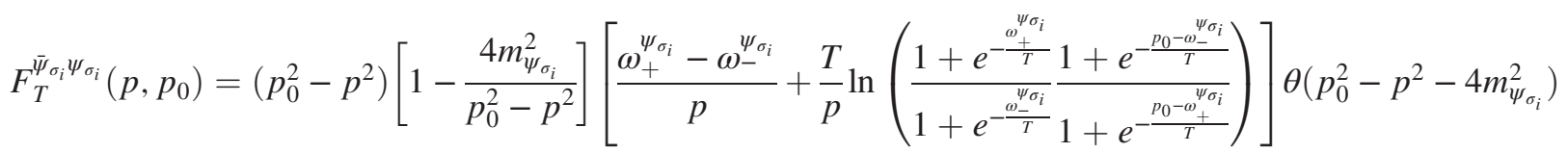

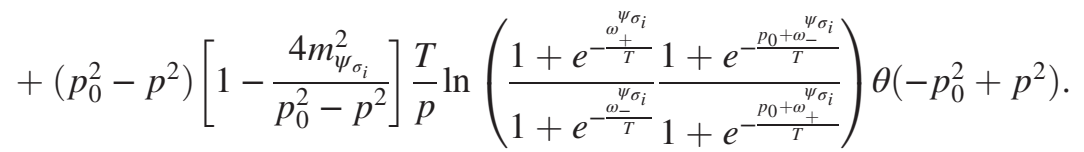


Then $m_{\psi_{\sigma_{i}}}$ is the fermionic $\psi_{\sigma_{i}}$ mass and

$$
\begin{aligned}
\omega_{ \pm}^{\psi_{\sigma_{i}}} & =\sqrt{\left(k_{ \pm}^{\psi_{\sigma_{i}}}\right)^{2}+m_{\psi_{\sigma_{i}}}^{2}}, \\
k_{ \pm}^{\psi_{\sigma_{i}}} & =\frac{1}{2}\left|p \pm p_{0}\left(1-\frac{4 m_{\psi_{\sigma_{i}}}^{2}}{p_{0}^{2}-p^{2}}\right)^{1 / 2}\right|
\end{aligned}
$$

An analysis of Eq. (A1) indicates that the behavior of the dissipation coefficient in the different temperature and interaction regimes is determined by an interplay between the spectral function and the thermal occupation numbers. When the temperature is large, the occupation numbers are also large, so in this regime, the poles of the spectral functions will dominate the integral, and this will be referred to as the pole approximation. As such, the pole approximation works well in the high- $T$ region. The dissipation coefficient about its pole at $p_{0} \simeq \omega_{p}$ is

$\Upsilon=\sum_{i=1}^{N_{M}} \frac{2}{T}\left(\frac{g^{2}}{2}\right)^{2}\left(\phi-M_{i}\right)^{2} \int \frac{d^{3} p}{(2 \pi)^{3}} \frac{n_{B}\left(1+n_{B}\right)}{\Gamma_{\chi_{i}} \omega_{p}^{2}}$.

For on-shell $\chi_{i}$ modes, decays into light scalars and fermions are equally probable, with partial decay widths given by Eqs. (A3) and (A6). By treating the scalar $\sigma_{i}$ and the fermion $\psi_{\sigma_{i}}$ as light fields, we evaluate both decay rates in the limit $m_{\sigma_{i}} \ll T\left(m_{\psi_{\sigma_{i}}} \ll T\right)$, so $\omega_{ \pm}^{\sigma_{i}} / T \simeq k_{ \pm}^{\sigma_{i}} / T\left(\omega_{ \pm}^{\psi_{\sigma_{i}}} / T \simeq\right.$ $\left.k_{ \pm}^{\psi_{\sigma_{i}}} / T\right)$; this estimation also yields that $k_{ \pm}^{\sigma_{i}}=k_{ \pm}^{\psi_{\sigma_{i}}}=$ $\left|p_{0} \pm p\right| / 2$. Finally, in the limit $m_{\chi_{i}} \ll T$, we have that both partial decay widths are given by

$$
\begin{gathered}
\Gamma_{\chi_{i}}\left(\chi_{i} \rightarrow \sigma_{i} \sigma_{i}\right)=\frac{h^{2}}{8 \pi} \frac{m_{\chi_{i}}^{2}}{\tilde{m}_{\chi_{i}}} \frac{T}{\omega_{p}}, \\
\Gamma_{\chi_{i}}\left(\chi_{i} \rightarrow \bar{\psi}_{\sigma_{i}} \psi_{\sigma_{i}}\right)=\frac{h^{2}}{128 \pi} \frac{\tilde{m}_{\chi_{i}}^{3}}{\omega_{p} T},
\end{gathered}
$$

yielding a total decay rate,

$$
\Gamma_{\chi_{i}}=\frac{h^{2}}{128 \pi} \frac{T \tilde{m}_{\chi_{i}}}{\omega_{\chi_{i}}(p)}\left[16 \frac{m_{\chi_{i}}^{2}}{\tilde{m}_{\chi_{i}}^{2}}+\frac{\tilde{m}_{\chi i}^{2}}{T^{2}}\right]
$$

Finally, we compute the integral in Eq. (A9) by following the same procedure as in [8], obtaining the total scalar dissipative coefficient,

$\Upsilon^{S}(\phi, T)=\sum_{i=1}^{N_{M}} \frac{32 g^{4}}{\pi h^{2}\left[16 \frac{m_{\chi_{i}}^{2}}{\tilde{m}_{\chi_{i}}^{2}}+\frac{\tilde{m}_{\chi_{i}}^{2}}{T^{2}}\right]} \ln \left(\frac{2 T}{\tilde{m}_{\chi_{i}}}\right) \frac{\left(\phi-M_{i}\right)^{2}}{\tilde{m}_{\chi_{i}}}$

\section{APPENDIX B: SCALAR DECAY WIDTH AVERAGE}

The thermal average of the scalar decay width is given by

$$
\bar{\Gamma}_{\chi_{i}}=\frac{1}{n_{B}} \int \frac{d^{3} p}{(2 \pi)^{3}} \Gamma_{\chi_{i}} f_{B},
$$

where $f_{B}(\mathbf{p} / T)$ is the Bose-Einstein distribution and $n_{B}\left(\tilde{m}_{\chi_{i}} / T\right)$ the associated number density. Hence we have

$$
\begin{aligned}
\bar{\Gamma}_{\chi_{i}} & =\frac{h^{2} T \tilde{m}_{\chi_{i}}}{128 \pi}\left[16 \frac{m_{\chi_{i}}^{2}}{\tilde{m}_{\chi_{i}}^{2}}+\frac{\tilde{m}_{\chi_{i}}^{2}}{T^{2}}\right] I\left(\tilde{m}_{\chi_{i}} / T\right), \\
I\left(\tilde{m}_{\chi_{i}} / T\right) & =\frac{\int \frac{d^{3} p}{(2 \pi)^{3}} \frac{f_{B}}{\omega_{\chi_{i}}(p)}}{\int \frac{d^{3} p}{(2 \pi)^{3}} f_{B}} .
\end{aligned}
$$

The integral factor $I\left(\tilde{m}_{\chi_{i}} / T\right)$ can be obtained numerically and is well approximated by

$I\left(\tilde{m}_{\chi_{i}} / T\right)=\frac{1}{T} \frac{\int \frac{d x x^{2}}{\sqrt{x^{2}+a^{2}}}\left(e^{\sqrt{x^{2}+a^{2}}}-1\right)^{-1}}{\int d x x^{2}\left(e^{\sqrt{x^{2}+a^{2}}}-1\right)^{-1}} \simeq \frac{1}{T} \frac{0.68}{(1+0.77 a)}$,

where we have defined $x=p / T$ and $a=\tilde{m}_{\chi_{i}} / T$. Hence the thermal average of the scalar decay width is

$$
\bar{\Gamma}_{\chi_{i}} \simeq \frac{h^{2} \tilde{m}_{\chi_{i}}}{128 \pi}\left[16 \frac{m_{\chi_{i}}^{2}}{\tilde{m}_{\chi_{i}}^{2}}+\frac{\tilde{m}_{\chi i}^{2}}{T^{2}}\right] \frac{0.68}{\left(1+0.77 f^{1 / 2}\right)} .
$$

Moreover, in the limit $m_{\chi_{i}} / T \lesssim 1$ we have that the effective finite thermal mass $\tilde{m}_{\chi_{i}}$ can fittingly be taken as $\tilde{m}_{\chi_{i}} \simeq f^{1 / 2} T$, where $f=f(g, h)=g^{2} / 12+h^{2} / 8$. Therefore the average decay width becomes

$$
\bar{\Gamma}_{\chi_{i}} \simeq \frac{h^{2} f^{1 / 2}}{128 \pi}\left[\frac{16}{f} \frac{m_{\chi_{i}}^{2}}{T^{2}}+f\right] \frac{0.68}{\left(1+0.77 f^{1 / 2}\right)} T .
$$

\section{APPENDIX C: EFFECTIVE POTENTIAL AT FINITE TEMPERATURE}

We start by considering the contribution of the light fermions in the tower to the finite temperature effective potential, which is given by [43-46]

$$
V_{T}^{\psi_{\chi_{i}}} \simeq \frac{1}{2} \sum_{i}\left\{-\frac{7 \pi^{2}}{180} T^{4}+\frac{\tilde{m}_{\psi_{\chi_{i}}}^{2} T^{2}}{12}+\frac{\tilde{m}_{\psi_{\chi_{i}}}^{4}}{16 \pi^{2}}\left[\ln \left(\frac{\mu^{2}}{T^{2}}\right)-c_{f}\right]\right\},
$$

where $\mu$ is the $\overline{\mathrm{MS}}$ renormalization scale, $c_{f}=2.635$, and the effective thermal fermion masses $\tilde{m}_{\psi i}^{2}=g^{2}\left(\phi-M_{i}\right)^{2}+$ $h^{2} T^{2} / 8$. Note that the overall factor of $1 / 2$ in front of the sum is related to the Majorana nature of the fermions in the SUSY model. In the continuum limit we may write this in the form 


$$
\begin{aligned}
V_{T}^{\psi_{i}} \simeq & \frac{T^{4}}{2} \sum_{i}\left\{-\frac{7 \pi^{2}}{180}+\left[\frac{g^{2}\left(\phi-M_{i}\right)^{2}}{T^{2}}+\frac{h^{2}}{8}\right] \frac{1}{12}+\left[\frac{g^{4}\left(\phi-M_{i}\right)^{4}}{T^{4}}+\frac{h^{2}}{4} \frac{g^{2}\left(\phi-M_{i}\right)^{2}}{T^{2}}+\frac{h^{4}}{64}\right] \frac{1}{16 \pi^{2}}\left[\ln \left(\frac{\mu^{2}}{T^{2}}\right)-c_{f}\right]\right\} \\
\simeq & \frac{T^{4}}{2}\left\{\left[-\frac{7 \pi^{2}}{180}+\frac{h^{2}}{96}+\frac{h^{4}}{1024 \pi^{2}}\left[\ln \left(\frac{\mu^{2}}{T^{2}}\right)-c_{f}\right]\right] \int_{M_{-}}^{M_{+}} d M n(M)+\frac{g^{2}}{T^{2}}\left[\frac{1}{12}+\frac{h^{2}}{64 \pi^{2}}\left[\ln \left(\frac{\mu^{2}}{T^{2}}\right)-c_{f}\right]\right] \int_{M_{-}}^{M_{+}} d M n(M)(\phi-M)^{2}\right. \\
& \left.+\frac{1}{16 \pi^{2}} \frac{g^{4}}{T^{4}}\left[\ln \left(\frac{\mu^{2}}{T^{2}}\right)-c_{f}\right] \int_{M_{-}}^{M_{+}} d M n(M)(\phi-M)^{4}\right\} \\
\simeq & \frac{T^{4}}{2}\left\{\left[-\frac{7 \pi^{2}}{180}+\frac{h^{2}}{96}+\frac{h^{4}}{1024 \pi^{2}}\left[\ln \left(\frac{\mu^{2}}{T^{2}}\right)-c_{f}\right]\right]\left[\frac{2 T}{g} n(\phi)+\cdots\right]+\frac{g^{2}}{T^{2}}\left[\frac{1}{12}+\frac{h^{2}}{64 \pi^{2}}\left[\ln \left(\frac{\mu^{2}}{T^{2}}\right)-c_{f}\right]\right]\left[\frac{2 T^{3}}{3}{ }^{3} n(\phi)+\cdots\right]\right. \\
& \left.+\frac{1}{16 \pi^{2}} \frac{g^{4}}{T^{4}}\left[\ln \left(\frac{\mu^{2}}{T^{2}}\right)-c_{f}\right]\left[\frac{2 T^{5}}{5} \frac{g^{5}}{g^{2}}(\phi)+\cdots\right]\right\} \\
\simeq & \frac{T^{5}}{2 g} n(\phi)\left\{-\frac{7 \pi^{2}}{90}+\frac{1}{18}+\frac{h^{2}}{48}+\frac{1}{16 \pi^{2}}\left(\frac{2}{5}+\frac{h^{2}}{6}+\frac{h^{4}}{32}\right)\left[\ln \left(\frac{\mu^{2}}{T^{2}}\right)-c_{f}\right]\right\}+\cdots,
\end{aligned}
$$

where the integrals are in general

$$
\begin{aligned}
\int_{M_{-}}^{M_{+}} d M n(M)(\phi-M)^{n} & =\int_{-T / g}^{T / g} d x\left[n(\phi)-x n^{\prime}(\phi)+\frac{x^{2}}{2 !} n^{\prime \prime}(\phi)+\cdots\right] x^{n} \\
& =n(\phi) \frac{x^{n+1}}{n+1}-n^{\prime}(\phi) \frac{x^{n+2}}{n+2}+n^{\prime \prime}(\phi) \frac{x^{n+3}}{n+3}+\left.\cdots\right|_{-T / g} ^{T / g} \\
& = \begin{cases}\frac{2}{n+1}\left(\frac{T}{g}\right)^{n+1} n(\phi)+\cdots, & n=0,2,4, \ldots \\
-\frac{2}{n+2}\left(\frac{T}{g}\right)^{n+2} n^{\prime}(\phi)+\cdots, & n=1,3,5, \ldots\end{cases}
\end{aligned}
$$

We have considered the local approximation, i.e., that only states in the vicinity of $M=\phi(t)$ are light at any given time. Note that to obtain the derivatives of this potential correction with respect to the field, one needs to take into account that both the integrand and the integration limits are $\phi$ dependent, the end result corresponding to differentiating Eq. (C2), such that

$$
\begin{gathered}
V_{T, \phi}^{\psi_{i}} \simeq \frac{T^{5}}{2 g} n^{\prime}(\phi)\left\{-\frac{7 \pi^{2}}{90}+\frac{1}{18}+\frac{h^{2}}{48}+\frac{1}{16 \pi^{2}}\left(\frac{2}{5}+\frac{h^{2}}{6}+\frac{h^{4}}{32}\right)\left[\ln \left(\frac{\mu^{2}}{T^{2}}\right)-c_{f}\right]\right\}+\cdots, \\
V_{T, \phi \phi}^{\psi_{i}} \simeq \frac{T^{5}}{2 g} n^{\prime \prime}(\phi)\left\{-\frac{7 \pi^{2}}{90}+\frac{1}{18}+\frac{h^{2}}{48}+\frac{1}{16 \pi^{2}}\left(\frac{2}{5}+\frac{h^{2}}{6}+\frac{h^{4}}{32}\right)\left[\ln \left(\frac{\mu^{2}}{T^{2}}\right)-c_{f}\right]\right\}+\cdots
\end{gathered}
$$

Nevertheless, in the local approximation the density of states could be taken only as temperature-dependent $n \sim T^{-1}$, and independent of the field; therefore $n^{\prime}(\phi)=n^{\prime \prime}(\phi)=0$. Thus we have $N_{M} \simeq 2 T n / g$, and hence $n \simeq g N_{M} /(2 T)$; but most importantly $V_{T, \phi}^{\psi_{\chi_{i}}}=V_{T, \phi \phi}^{\psi_{i}} \simeq 0$. Finally, the finite temperature corrections to the effective potential due to the fermionic tower, in the local approximation at leading order, can be written as

$$
V_{T}^{\psi_{i}} \simeq \frac{N_{M}}{4} T^{4}\left\{-\frac{7 \pi^{2}}{90}+\frac{1}{18}+\frac{h^{2}}{48}+\frac{1}{16 \pi^{2}}\left(\frac{2}{5}+\frac{h^{2}}{6}+\frac{h^{4}}{32}\right)\left[\ln \left(\frac{\mu^{2}}{T^{2}}\right)-c_{f}\right]\right\} .
$$

Next we examine the contribution of the light bosons in the tower to the finite temperature effective potential given by [43-46]

$$
V_{T}^{\chi_{i}} \simeq 2 \sum_{i}\left\{-\frac{\pi^{2}}{90} T^{4}+\frac{\tilde{m}_{\chi_{i}}^{2} T^{2}}{24}-\frac{\tilde{m}_{\chi_{i}}^{3} T}{12 \pi}-\frac{\tilde{m}_{\chi_{i}}^{4}}{64 \pi^{2}}\left[\ln \left(\frac{\mu^{2}}{T^{2}}\right)-c_{b}\right]\right\}
$$


where again $\mu$ denotes the $\overline{\mathrm{MS}}$ renormalization scale, $c_{b}=5.41$, and the effective thermal boson masses $\tilde{m}_{\chi_{i}}^{2}=g^{2}\left(\phi-M_{i}\right)^{2}+g^{2} T^{2} / 12+h^{2} T^{2} / 8$. Note the overall factor of 2 in front of the sum, which represents the fact that the $\chi_{i}$ 's scalars are complex fields. Following the same procedure as for the fermionic tower, we have for the bosonic sector

$$
\begin{aligned}
V_{T}^{\chi_{i}} \simeq & \frac{2 T^{5}}{g} n(\phi)\left\{-\frac{\pi^{2}}{45}+\frac{f^{2}(g, h)}{16 \pi} \ln \left(\frac{\sqrt{f(g, h)}}{\sqrt{1+f(g, h)}+1}\right)+\frac{f(g, h)}{12}-\frac{(2+5 f(g, h))}{48 \pi} \sqrt{1+f(g, h)}\right. \\
& \left.-\frac{1}{16 \pi^{2}}\left(\frac{1}{10}+\frac{f(g, h)}{3}+\frac{f^{2}(g, h)}{2}\right)\left[\ln \left(\frac{\mu^{2}}{T^{2}}\right)-c_{b}\right]\right\}+\cdots,
\end{aligned}
$$

where $f(g, h)=g^{2} / 12+h^{2} / 8$. In the local approximation $n(\phi) \sim T^{-1}$, hence $n(\phi) \simeq g N_{\chi} /(2 T)$, and the inflaton $\phi$ derivatives are zero: $V_{T, \phi}^{\chi_{i}}=V_{T, \phi \phi}^{\chi_{i}} \simeq 0$. Finally, the finite temperature corrections to the effective potential due to the bosonic tower, in the local approximation at leading order, can be written in the form

$$
\begin{aligned}
V_{T}^{\chi_{i}} \simeq & N_{M} T^{4}\left\{-\frac{\pi^{2}}{45}+\frac{f^{2}(g, h)}{16 \pi} \ln \left(\frac{\sqrt{f(g, h)}}{\sqrt{1+f(g, h)}+1}\right)+\frac{f(g, h)}{12}-\frac{(2+5 f(g, h))}{48 \pi} \sqrt{1+f(g, h)}\right. \\
& \left.-\frac{1}{16 \pi^{2}}\left(\frac{1}{10}+\frac{f(g, h)}{3}+\frac{f^{2}(g, h)}{2}\right)\left[\ln \left(\frac{\mu^{2}}{T^{2}}\right)-c_{b}\right]\right\} .
\end{aligned}
$$

[1] P. A. R. Ade et al. (Planck Collaboration), arXiv: 1807.06211.

[2] A. A. Starobinsky, Phys. Lett. 91B, 99 (1980); K. Sato, Mon. Not. R. Astron. Soc. 195, 467 (1981); A. H. Guth, Phys. Rev. D 23, 347 (1981); A. Albrecht and P. J. Steinhardt, Phys. Rev. Lett. 48, 1220 (1982); A. D. Linde, Phys. Lett. B 108, 389 (1982).

[3] A. Albrecht, P. J. Steinhardt, M. S. Turner, and F. Wilczek, Phys. Rev. Lett. 48, 1437 (1982); L. F. Abbott, E. Farhi, and M. B. Wise, Phys. Lett. 117B, 29 (1982); L. Kofman, A. D. Linde, and A. A. Starobinsky, Phys. Rev. D 56, 3258 (1997); P. B. Greene, L. Kofman, A. D. Linde, and A. A. Starobinsky, Phys. Rev. D 56, 6175 (1997).

[4] I. G. Moss and C. M. Graham, Phys. Rev. D 78, 123526 (2008).

[5] A. Berera and L.-Z. Fang, Phys. Rev. Lett. 74, 1912 (1995).

[6] A. Berera, Phys. Rev. Lett. 75, 3218 (1995).

[7] A. Berera, Phys. Rev. D 54, 2519 (1996).

[8] A. Berera, M. Gleiser, and R. O. Ramos, Phys. Rev. D 58, 123508 (1998).

[9] A. Berera and T. W. Kephart, Phys. Rev. Lett. 83, 1084 (1999).

[10] A. Berera, Nucl. Phys. B585, 666 (2000).

[11] A. Berera, M. Gleiser, and R. O. Ramos, Phys. Rev. Lett. 83, 264 (1999).

[12] M. R. Douglas, Lecture at JHS60 (California Institute of Technology, Pasadena, California, USA, 2001).

[13] M. R. Douglas, J. High Energy Phys. 05 (2003) 046.

[14] C. Vafa, arXiv:hep-th/0509212.

[15] H. Ooguri and C. Vafa, Nucl. Phys. B766, 21 (2007).
[16] G. Obied, H. Ooguri, L. Spodyneiko, and C. Vafa, arXiv: 1806.08362.

[17] P. Agrawal, G. Obied, P. J. Steinhardt, and C. Vafa, Phys. Lett. B 784, 271 (2018).

[18] S. Das, arXiv:1809.03962.

[19] S. Das, Phys. Rev. D 99, 063514 (2019).

[20] M. Motaharfar, V. Kamali, and R. O. Ramos, Phys. Rev. D 99, 063513 (2019).

[21] Z. Yi and Y. Gong, arXiv:1811.01625.

[22] W. C. Lin and W. H. Kinney, arXiv:1812.04447.

[23] A. Berera, I. G. Moss, and R. O. Ramos, Rep. Prog. Phys. 72, 026901 (2009).

[24] A. Berera, Phys. Rev. D 55, 3346 (1997).

[25] M. Bastero-Gil and A. Berera, Int. J. Mod. Phys. A 24, 2207 (2009).

[26] Ø. Grøn, Universe 2, 20 (2016).

[27] R. Rangarajan, arXiv:1801.02648.

[28] L. M. Hall, I. G. Moss, and A. Berera, Phys. Rev. D 69 , 083525 (2004).

[29] I. G. Moss and C. Xiong, J. Cosmol. Astropart. Phys. 04 (2007) 007.

[30] R. O. Ramos and L. A. da Silva, J. Cosmol. Astropart. Phys. 03 (2013) 032.

[31] M. Bastero-Gil, A. Berera, and R. O. Ramos, J. Cosmol. Astropart. Phys. 07 (2011) 030.

[32] Y. F. Cai, J. B. Dent, and D. A. Easson, Phys. Rev. D 83, 101301 (2011).

[33] S. Bartrum, M. Bastero-Gil, A. Berera, R. Cerezo, R. O. Ramos, and J. G. Rosa, Phys. Lett. B 732, 116 (2014). 
[34] M. Bastero-Gil, A. Berera, R. O. Ramos, and J. G. Rosa, Phys. Rev. Lett. 117, 151301 (2016).

[35] M. Bastero-Gil, S. Bhattacharya, K. Dutta, and M. R. Gangopadhyay, J. Cosmol. Astropart. Phys. 02 (2018) 054.

[36] M. Bastero-Gil, A. Berera, R. Hernández-Jiménez, and J. G. Rosa, Phys. Rev. D 98, 083502 (2018).

[37] F. L. Bezrukov and M. Shaposhnikov, Phys. Lett. B 659, 703 (2008).

[38] L. M. H. Hall and I. G. Moss, Phys. Rev. D 71, 023514 (2005).

[39] M. Bastero-Gil, A. Berera, and R. O. Ramos, J. Cosmol. Astropart. Phys. 09 (2011) 033.

[40] M. Bastero-Gil, A. Berera, R. O. Ramos, and J. G. Rosa, J. Cosmol. Astropart. Phys. 01 (2013) 016.

[41] We basically take the decay widths of the processes $\chi_{i} \rightarrow$ $\bar{\psi}_{\sigma_{i}} \psi_{\sigma_{i}}$ (eqs. (B.12)) and $\psi_{\chi_{i}} \rightarrow \sigma_{i} \psi_{\sigma_{i}}$ (eqs. (B.17-B.20)) from [39]. We employ the pole approximation $\left(p_{0}=\omega_{X}(p) \simeq m_{\chi_{i}}\right)$ and take $T \rightarrow 0$ in order to identify the vertex factor squared.

[42] A. Berera and T.W. Kephart, Phys. Lett. B 456, 135 (1999).

[43] L. Dolan and R. Jackiw, Phys. Rev. D 9, 3320 (1974).
[44] J. I. Kapusta and C. Gale, Finite-Temperature Field Theory: Principles and Applications (Cambridge University Press, Cambridge, England, 2006).

[45] J. M. Cline and P. A. Lemieux, Phys. Rev. D 55, 3873 (1997).

[46] G. W. Anderson and L. J. Hall, Phys. Rev. D 45, 2685 (1992).

[47] C. Graham and I. G. Moss, J. Cosmol. Astropart. Phys. 07 (2009) 013.

[48] M. Bastero-Gil, A. Berera, I. G. Moss, and R. O. Ramos, J. Cosmol. Astropart. Phys. 05 (2014) 004.

[49] S. Bartrum, A. Berera, and J. G. Rosa, J. Cosmol. Astropart. Phys. 06 (2013) 025.

[50] M. Kamionkowski and E. D. Kovetz, Annu. Rev. Astron. Astrophys. 54, 227 (2016).

[51] A. Kehagias and A. Riotto, Fortschr. Phys. 66, 1800052 (2018).

[52] Y. Akrami, R. Kallosh, A. Linde, and V. Vardanyan, Fortschr. Phys. 67, 1800075 (2019).

[53] J. G. Rosa and L. B. Ventura, Phys. Rev. Lett. 122, 161301 (2019).

[54] R. Arya and R. Rangarajan, arXiv:1812.03107.

[55] A. Berera, Proc. Sci., AHEP2003 (2003) 069.

[56] R. Kallosh and A. Linde, J. Cosmol. Astropart. Phys. 11 (2010) 011. 